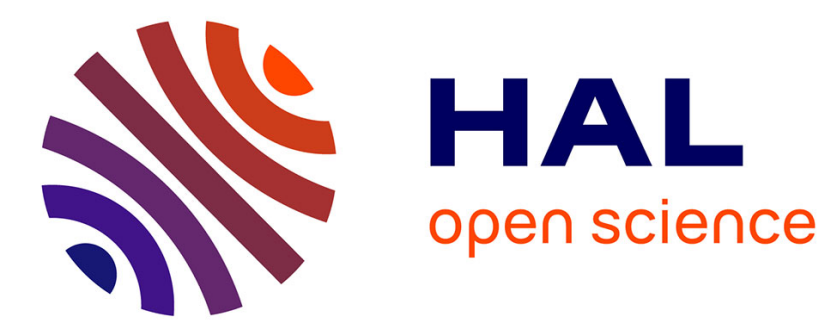

\title{
Extracting generalized edge flux intensity functions with the quasidual function method along circular 3-D edges
}

\author{
Samuel Shannon, Zohar Yosibash, Monique Dauge, Martin Costabel
}

\section{To cite this version:}

Samuel Shannon, Zohar Yosibash, Monique Dauge, Martin Costabel. Extracting generalized edge flux intensity functions with the quasidual function method along circular 3-D edges. International Journal of Fracture, 2013, 181 (1), pp.25-50. 10.1007/s10704-013-9817-4 . hal-00725928v2

\section{HAL Id: hal-00725928 \\ https://hal.science/hal-00725928v2}

Submitted on 18 Dec 2012

HAL is a multi-disciplinary open access archive for the deposit and dissemination of scientific research documents, whether they are published or not. The documents may come from teaching and research institutions in France or abroad, or from public or private research centers.
L'archive ouverte pluridisciplinaire HAL, est destinée au dépôt et à la diffusion de documents scientifiques de niveau recherche, publiés ou non, émanant des établissements d'enseignement et de recherche français ou étrangers, des laboratoires publics ou privés. 


\title{
EXTRACTING GENERALIZED EDGE FLUX INTENSITY FUNCTIONS BY THE QUASIDUAL FUNCTION METHOD ALONG CIRCULAR 3-D EDGES
}

\author{
SAMUEL SHANNON, ZOHAR YOSIBASH, MONIQUE DAUGE AND MARTIN COSTABEL
}

\begin{abstract}
Explicit asymptotic series describing solutions to the Laplace equation in the vicinity of a circular edge in a three-dimensional domain was recently provided in Yosibash et al, Int. Jour. Fracture, 168 (2011), pp. 3152. Utilizing it, we extend the quasidual function method (QDFM) for extracting the generalized edge flux intensity functions (GEFIFs) along circular singular edges in the cases of axisymmetric and non-axisymmetric data.

This accurate and efficient method provides a functional approximation of the GEFIFs along the circular edge whose order is adaptively increased so to approximate the exact GEFIFs. It is implemented as a post-solution operation in conjunction with the $p$-version of the finite element method. The mathematical analysis of the QDFM is provided, followed by numerical investigations, demonstrating the efficiency, robustness and high accuracy of the proposed quasi-dual function method. The mathematical machinery developed in the framework of the Laplace operator is important to realize its possible extension for the elasticity system.
\end{abstract}

\section{CONTENTS}

1. Introduction

2. A Path-Independent Integral Around a Corner in 2-D 2

3. The Surface-Integral $J_{\rho}\left[\tau, K_{n, m}^{\left(\alpha_{j}\right)}\right]$ around a Circular Edge in 3-D 4

3.1. Local coordinates around a circular edge 4

3.2. Primal singular functions and their shadows $\quad 5$

3.3. Dual singular functions, their shadows, and the surface-integral $\quad 6$

3.4. Extracting circular GEFIFs using the quasidual-function method 8

4. Extracting Circular GEFIFs for Axisymmetric Solutions by the QDFM 11

4.1. A circular crack with homogenous Neumann BCs - Axisymmetric case 12

4.2. Axisymmetric solution of a $3 \pi / 2$ V-notch with homogeneous Neumann BCs 15

4.3. Extracting $A_{j}$ from $p$-FE solutions 17

5. Nonaxisymmetric solutions 23

5.1. $B_{j}(\theta)$ for a nonaxisymmetric case 23

5.2. A circular crack with homogeneous Neumann BCs 24

5.3. A $3 \pi / 2$ V-notch with homogeneous Neumann BCs 26

5.4. Extracting $a_{j_{q}}(\theta)$ from $p$-FE nonaxisymmetric solutions 27

6. Summary and conclusions 31

References $\quad 31$

Appendix A. An explicit expression for $K_{\infty, 0}^{(1 / 2)}$ for a circular crack $\quad 33$

Appendix B. Tables with results of the surface integral in a non-axisymmetric case 34

\section{INTRODUCTION}

Methods for computing stress intensity factors (SIFs) for crack tips and generalized SIFs (GSIFs) for Vnotch tips in two-dimensional (2-D) domains were addressed in many papers in the past five decades, starting with $[8,9]$. In realistic three-dimensional domains however, edge singularities (crack fronts and V-notch tip 
curves) attracted much scarcer attention due to the complexity of the solution in the vicinity of edges (except a pioneering work as early as 1946, by Sneddon [13], for the penny-shaped crack in an infinite domain). The SIFs and GSIFs are variable along such edges, defining univariate functions called edge stress intensity functions (ESIFs) and generalized edge stress intensity functions (GESIFs).

In the vicinity of straight and circular edges an explicit asymptotic series of the singular solutions was provided $[5,11,14,15]$. Each term of this series is characterized by:

- an exponent $\alpha_{k}$ which belongs to a discrete set $\left\{\alpha_{k}, k \in \mathbb{N}\right\}$ of eigenvalues depending only on the geometry and the operator, and which determines the level of non-smoothness of the singularity. Any eigenvalue $\alpha_{k}$ is computed by solving a 1-D problem.

- a generalized eigenfunction expansion $\phi_{k, j, \ell}(\varphi)(\varphi$ is an angular coordinate transverse to the edge) which depends on the geometry of the domain and the operator. The terms of this expansion are computed by solving a set of 1-D problems.

- a function along the edge, denoted by $A_{k}(\theta)$ ( $\theta$ is a coordinate along the edge) and called "generalized edge flux/stress intensity function" (GEFIF/GESIF) which determines the "amount of energy" residing in each singularity.

Based on the explicit representation of the solution to the Laplace and elasticity system in the vicinity of a circular edge in [15], here we extend the quasidual function method (QDFM) presented in the framework of 3 -D straight edges in $[5,11]$ to circular edges. This extension is demonstrated on the basis of the the Laplace equation. This is because it is a simpler elliptic operator that allows more transparent analytic computations and invokes all necessary characteristics of the elasticity system. Thus, the characteristics of the QDFM for circular edges can be more easily addressed so to be extended thereafter to the elasticity system for the computation of ESIFs for cracks occurring usually in pipes and pressure vessels.

We construct the quasidual functions (QDF) $K_{n, m}^{\left(\alpha_{j}\right)}$ adapted to circular edges. They are the essential ingre-

dient used to define a new functional, $\tau \mapsto J_{\rho_{0}}\left[\tau, K_{n, m}^{\left(\alpha_{j}\right)}\right]$, which is a surface integral on a torus of minor radius $\rho_{0}$ having the singular edge as its axis. The result of this functional determines an explicit representation of the GEFIF of the solution $\tau$ in a certain basis of functions, as opposed to other methods providing pointwise values along the edge. Since the GEFIF on a circular edge is a periodic function, it will be natural to construct the QDFs in such a way that the functional determines the Fourier coefficients of the GEFIF. Furthermore, the new method allows to extract the GEFIFs away from the singular edge, thus enables the use of coarse meshes and alleviates the necessity of complex refined mesh generation in the vicinity of 3-D singular edges. The obtained results are both accurate, efficient and robust.

Notation and preliminaries are introduced in section 2 and the dual-function method in 2-D domains, known also as the contour-integral method [2, 1], is recalled. It serves as the basis to the QDFM. In section 3 we extend the QDFM to circular domains by providing a mathematical analysis on its theoretical performance. It is then used in section 4 to extract the GEFIFs from axisymmetric solutions along circular cracks and circular V-notch edges. Our method can be compared with the method of the singular complement of [3]. Numerical examples using the $p$-version of the finite element method are also provided to demonstrate the efficiency of the QDFM in practical applications. Circular edges in nonaxisymmetric cases are addressed in section 5, and we summarize our results in section 6.

\section{A Path-Independent Integral Around a Corner in 2-D}

The Laplace equation in a 2-D domain expressed in polar coordinates $(\rho, \varphi)$ located in the singular point $P$ (see Figure 1) is given by

$$
\Delta \tau=\frac{1}{\rho^{2}}\left[\left(\rho \partial_{\rho}\right)^{2}+\partial_{\varphi \varphi}\right] \tau=0 \quad \text { in } \Omega
$$


with either Dirichlet or Neumann homogeneous boundary conditions (BCs) on the faces $\Gamma_{1}$ and $\Gamma_{2}(\varphi=$ $\left.\varphi_{1}, \varphi_{2}\right)$ intersecting at $P$,

$$
\begin{aligned}
\tau & =0 \text { on } \Gamma_{1} \cup \Gamma_{2} \text { Drichlet BCs } \\
\frac{\partial \tau}{\partial \hat{n}}=\nabla \tau \cdot \hat{\boldsymbol{n}} & =0 \text { on } \Gamma_{1} \cup \Gamma_{2} \text { Neumann BCs }
\end{aligned}
$$

where $\hat{\boldsymbol{n}}$ is the outward normal vector. Solutions to (1) with (2) or (3) expand in the vicinity of $P$ as asymp-

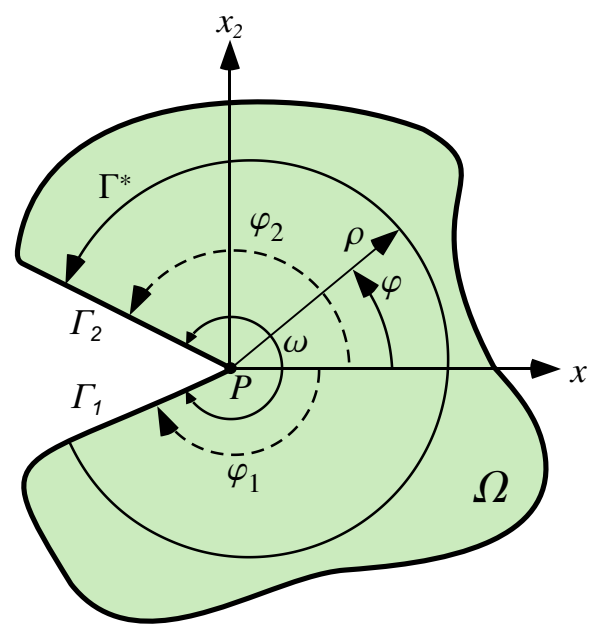

FIGURE 1. 2-D domain and notations.

totic series composed of primal eigenfunctions and GFIFs

$$
\tau=\sum_{k \geq 0} A_{k} \rho^{\alpha_{k}} \phi_{k}(\varphi)
$$

where both the eigenvalues $\alpha_{k}$ and eigenfunctions $\phi_{k}(\varphi)$ may be, for the Laplace equation, explicitly computed [7]. They can be expressed as follows

$$
\alpha_{k}=\frac{k \pi}{\omega}
$$

and

$$
\phi_{k}(\varphi)= \begin{cases}\rho^{\frac{k \pi}{\omega}} \sin \frac{k \pi}{\omega}\left(\varphi-\varphi_{1}\right) & \text { (Dirichlet) } \\ \rho^{\frac{k \pi}{\omega}} \cos \frac{k \pi}{\omega}\left(\varphi-\varphi_{1}\right) & \text { (Neumann). }\end{cases}
$$

Each primal eigenpair is associated with a dual eigenpair $\left(-\alpha_{k}, \psi_{k}(\varphi)\right)$, that satisfies the PDE and BCs but does not belong to the energy space (equivalent to the $H^{1}(\Omega)$ Sobolev space). For the Laplace equation $\psi_{k}(\varphi) \equiv \phi_{k}(\varphi)$. These dual eigenpairs are used to construct dual singular functions

$$
K^{\left(\alpha_{j}\right)} \stackrel{\text { def }}{=} B_{j} \rho^{-\alpha_{j}} \psi_{j}(\varphi)
$$

where:

$$
B_{j}=\frac{1}{2 \alpha_{j} D_{j}}
$$

with the constant $D_{j}$ defined as the scalar product of the angular functions:

$$
D_{j}=\int_{\varphi_{1}}^{\varphi_{2}} \phi_{j}(\varphi) \psi_{j}(\varphi) d \varphi=\int_{\varphi_{1}}^{\varphi_{2}} \phi_{j}^{2}(\varphi) d \varphi
$$


The dual-function method, known also as the contour-integral method, is very efficient for the extraction of the generalized flux intensity factors (GFIFs) utilizing a path-independent integral $[2,1]$ along a path starting on $\Gamma_{1}$ and terminating on $\Gamma_{2}$ :

$$
J_{\Gamma^{*}}\left[\tau, K^{\left(\alpha_{j}\right)}\right] \stackrel{\text { def }}{=} \oint_{\Gamma^{*}}\left(K^{\left(\alpha_{j}\right)} \partial_{\rho} \tau-\tau \partial_{\rho} K^{\left(\alpha_{j}\right)}\right) d \Gamma=A_{j} .
$$

Here $d \Gamma=\rho d \varphi$. Furthermore, $J_{\Gamma^{*}}\left[\tau, K^{\left(\alpha_{j}\right)}\right]$ is path-independent.

\section{The Surface-Integral $J_{\rho}\left[\tau, K_{n, m}^{\left(\alpha_{j}\right)}\right]$ ARound a Circular Edge in 3-D}

3.1. Local coordinates around a circular edge. Consider a circular singular edge in a 3-D domain, generated by rotating the singular point $P$ around the $x_{3}$ axis as shown in Figure 2. Cylindrical coordinates are $\left(r, \theta, x_{3}\right)$ with the distance $r$ to the axis and the rotation angle $\theta$. In general, neither the domain, nor the boundary conditions have to be axisymmetric, but for ease of presentation we consider the 3-D domain generated by the cross-section $\Omega$. Locating a polar coordinate system $(\rho, \varphi)$ at $P$, we have the relations

$$
r=R+\rho \cos \varphi \quad \text { and } \quad x_{3}=\rho \sin \varphi .
$$

and the Laplace operator can be written in $(\rho, \varphi, \theta)$ coordinates as (see [15]):

$$
\left\{\begin{aligned}
& \Delta= \frac{1}{\rho^{2}} \frac{1}{\left(1+\frac{\rho}{R} \cos \varphi\right)^{2}} \widetilde{\Delta}\left(\varphi ; \partial_{\varphi}, \rho \partial_{\rho}, \partial_{\theta}\right) \text { with } \\
& \widetilde{\Delta} \stackrel{\text { def }}{=}\left(1+\frac{\rho}{R} \cos \varphi\right)^{2}\left[\left(\rho \partial_{\rho}\right)^{2}+\partial_{\varphi \varphi}\right] \\
& \quad+\left(1+\frac{\rho}{R} \cos \varphi\right) \times\left(\frac{\rho}{R}\right)\left[\cos \varphi \rho \partial_{\rho}-\sin \varphi \partial_{\varphi}\right]+\left(\frac{\rho}{R}\right)^{2} \partial_{\theta \theta}
\end{aligned}\right.
$$

In the vicinity of the circular edge let the function $\tau$ satisfy

$$
\Delta \tau=0, \quad \text { i.e. } \widetilde{\Delta}\left(\varphi ; \partial_{\varphi}, \rho \partial_{\rho}, \partial_{\theta}\right) \tau(\varphi, \rho, \theta)=0,
$$

with homogeneous Dirichlet or Neumann BCs on the re-entrant faces

$$
\begin{aligned}
& \tau=0 \text { on } \varphi=\varphi_{1}, \varphi=\varphi_{2} \text {, Dirichlet BCs } \\
& \nabla \tau \cdot \hat{n}=0 \text { on } \varphi=\varphi_{1}, \varphi=\varphi_{2} \text {, Neumann BCs }
\end{aligned}
$$

The interior equation (10) with (11) or (12) is completed by (non-zero) regular boundary conditions on the rest of the boundary of $\Omega$.

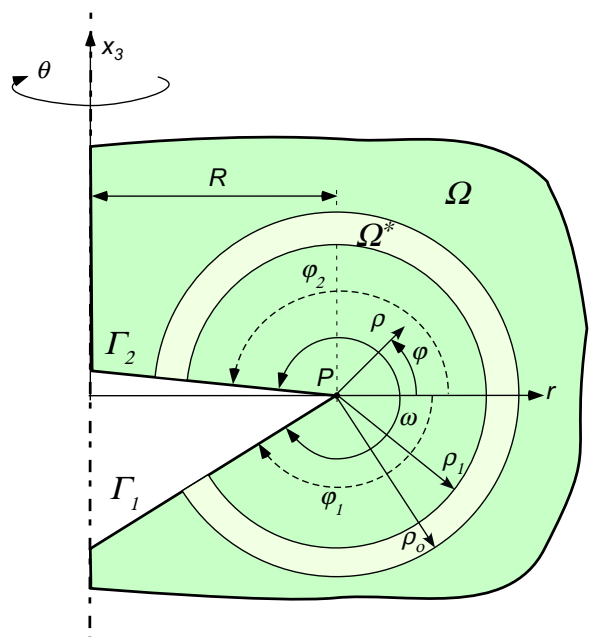

FIGURE 2. 3-D domain of interest $\Omega$ and the $(\rho, \varphi, \theta)$ coordinate system. 
3.2. Primal singular functions and their shadows. According to [10], solutions $\tau$ to the 3-D problem (10) with BCs (11) or (12) expand as asymptotic series in powers of $\rho$ and $\log \rho$ as $\rho \rightarrow 0$. The structure of this asymptotic series is driven by the principal part $\Delta^{\text {prcp }}$ of the operator $\Delta$, frozen along the edge. Using (9), we find $\Delta^{\text {prcp }}=\rho^{-2}\left[\left(\rho \partial_{\rho}\right)^{2}+\partial_{\varphi \varphi}\right]$. As a consequence, the 3-D asymptotic series is generated by the 2-D eigenpairs $\alpha_{k}$ and $\phi_{k}$ presented in Section 2. Due to the regularity of data, the 3-D asymptotic series takes the general form of a sum in $k \geq 1$ of singular packets

$$
\tau(\varphi, \rho, \theta) \underset{\rho \rightarrow 0}{\simeq} \sum_{k \geq 0} A_{k}(\theta) \rho^{\alpha_{k}} \phi_{0, k, 0}(\varphi)+\mathfrak{Z}_{k}\left[A_{k}\right](\varphi, \rho, \theta) .
$$

The functions $\phi_{0, k, 0} \stackrel{\text { def }}{=} \phi_{k}$ are called primal (singular) functions. Each of the higher order terms $\mathfrak{Z}_{k}$ has a composite structure involving so-called shadow functions. Since the operator $\widetilde{\Delta}$ has constant coefficients with respect to the variable $\theta$ and involves no first-order derivative with respect to $\theta$, the general form appearing in [10] simplifies. In the non-resonant case (i.e. when $\alpha_{k}-\alpha_{j}$ is never integer) $\mathfrak{Z}_{k}$ involves even-order derivatives of $A_{k}$ and a two-parameter family of shadow functions: $\phi_{\ell, k, i}$ for $\ell=0,2,4, \ldots$ and $i=$ $0,1,2, \ldots$

$$
\forall k \geq 1, \quad \mathfrak{Z}_{k}\left[A_{k}\right](\varphi, \rho, \theta) \underset{\rho \rightarrow 0}{\simeq} \underbrace{\sum_{\ell=0,2,4, \cdots} \sum_{i \geq 0}}_{\ell+i>0} \partial_{\theta}^{\ell} A_{k}(\theta) \rho^{\alpha_{k}}\left(\frac{\rho}{R}\right)^{i+\ell} \phi_{\ell, k, i}(\varphi) .
$$

Fortunately, the important case of cracks, which is a priori resonant, enters also in this framework by virtue of the absence of logarithmic terms, [4]. Thus we write

$$
\tau=\sum_{k \geq 0} \sum_{\ell=0,2,4, \cdots} \partial_{\theta}^{\ell} A_{k}(\theta) \rho^{\alpha_{k}} \sum_{i \geq 0}\left(\frac{\rho}{R}\right)^{i+\ell} \phi_{\ell, k, i}(\varphi)
$$

where $A_{k}(\theta)$ are the generalized edge flux intensity functions (GEFIFs).

Remark 1. The explicit ODEs for the determination of $\phi_{\ell, k, i}$ provided in [15] are written in a more abstract form here for the purpose of mathematical proofs (where we also introduce many notations and indices local to this section).

The generalized relations satisfied by all $\phi_{\ell, k, i}$ may be described with the help of the expansion of the Laplace operator $\widetilde{\Delta}$ defined in (9) with respect to powers of $\frac{\rho}{R}$ :

$$
\widetilde{\Delta}=\sum_{\ell=0}^{2} \sum_{i=0}^{2}\left(\frac{\rho}{R}\right)^{i+\ell} \partial_{\theta}^{\ell} M_{\ell, i}\left(\varphi ; \partial_{\varphi}, \rho \partial_{\rho}\right)
$$

where the $M_{\ell, i}$ are partial differential operators of order $\leq 2$, with coefficients independent of $\rho$. Explicitly we have (note that $M_{0,0}=\rho^{2} \Delta^{\text {prcp }}$ )

$$
\left\{\begin{array}{l}
M_{0,0}=\left(\rho \partial_{\rho}\right)^{2}+\partial_{\varphi \varphi} \\
M_{0,1}=2 \cos \varphi\left[\left(\rho \partial_{\rho}\right)^{2}+\frac{1}{2}\left(\rho \partial_{\rho}\right)+\partial_{\varphi \varphi}\right]-\sin \varphi \partial_{\varphi} \\
M_{0,2}=\cos ^{2} \varphi\left[\left(\rho \partial_{\rho}\right)^{2}+\left(\rho \partial_{\rho}\right)+\partial_{\varphi \varphi}\right]-\frac{1}{2} \sin 2 \varphi \partial_{\varphi}, \\
M_{1, i}=0, i=0,1,2, \quad M_{2,0}=1, M_{2,1}=0, M_{2,2}=0 .
\end{array}\right.
$$

For each $k$, the equations that $\phi_{\ell, k, i}$ satisfy are found relying on the fact that each singular packet in (13)

$$
A_{k}(\theta) \rho^{\alpha_{k}} \phi_{0, k, 0}(\varphi)+\mathfrak{Z}_{k}\left[A_{k}\right](\varphi, \rho, \theta)
$$

is a formal solution of (10), for any coefficient $A_{k}$. Thus we find for each $k \geq 1$

$$
\sum_{\ell=0}^{2} \sum_{i=0}^{2}\left(\frac{\rho}{R}\right)^{i+\ell} \partial_{\theta}^{\ell} M_{\ell, i}\left(\varphi ; \partial_{\varphi}, \rho \partial_{\rho}\right) \sum_{\ell^{\prime} \geq 0} \sum_{i^{\prime} \geq 0} \partial_{\theta}^{\ell^{\prime}} A_{k}(\theta) \rho^{\alpha_{k}}\left(\frac{\rho}{R}\right)^{i^{\prime}+\ell^{\prime}} \phi_{\ell^{\prime}, k, i^{\prime}}(\varphi)=0 .
$$


Using the relation

$$
M_{\ell, i}\left(\varphi ; \partial_{\varphi}, \rho \partial_{\rho}\right) \rho^{\alpha_{k}}\left(\frac{\rho}{R}\right)^{i^{\prime}+\ell^{\prime}}=\rho^{\alpha_{k}}\left(\frac{\rho}{R}\right)^{i^{\prime}+\ell^{\prime}} M_{\ell, i}\left(\alpha_{k}+\ell^{\prime}+i^{\prime}\right)
$$

where $M_{\ell, i}(\beta)$ is a shorthand for $M_{\ell, i}\left(\varphi ; \partial_{\varphi}, \beta\right)$, equation (18) becomes

$$
\rho^{\alpha_{k}} \sum_{\ell=0}^{2} \sum_{i=0}^{2} \sum_{\ell^{\prime} \geq 0} \sum_{i^{\prime} \geq 0}\left(\frac{\rho}{R}\right)^{i+\ell+i^{\prime}+\ell^{\prime}} \partial_{\theta}^{\ell+\ell^{\prime}} A_{k}(\theta) M_{\ell, i}\left(\alpha_{k}+\ell^{\prime}+i^{\prime}\right) \phi_{\ell^{\prime}, k, i^{\prime}}(\varphi)=0 .
$$

Setting $\lambda=\ell+\ell^{\prime}$ and $\mu=i+i^{\prime}$, we find the equivalent relation

$$
\sum_{\lambda \geq 0} \sum_{\mu \geq 0} \sum_{\ell=0}^{2} \sum_{i=0}^{2}\left(\frac{\rho}{R}\right)^{\lambda+\mu} \partial_{\theta}^{\lambda} A_{k}(\theta) M_{\ell, i}\left(\alpha_{k}+\lambda-\ell+\mu-i\right) \phi_{\lambda-\ell, k, \mu-i}(\varphi)=0 .
$$

This relation holds for any function $A_{k}(\theta)$ and for all $\rho>0$ if and only if

$$
\sum_{\ell=0}^{2} \sum_{i=0}^{2} M_{\ell, i}\left(\alpha_{k}+\lambda-\ell+\mu-i\right) \phi_{\lambda-\ell, k, \mu-i}(\varphi)=0, \quad \forall \lambda, \mu \geq 0
$$

Equation (21) is the key for the recursive construction of the shadow terms (the explicit recursive equations are given in [15]). In [12] we provide formulas for the functions $\phi_{\ell, k, i}(\varphi)$ in the case of a penny-shaped crack and a $90^{\circ}$ V-notch with Neumann BCs.

3.3. Dual singular functions, their shadows, and the surface-integral. To extract the generalized edge flux intensity functions $A_{k}(\theta)$, we extend the path independent-integral presented in the previous section.

Similar to the primal functions and their shadows, for any $j \geq 1$, we introduce the dual function $\psi_{0, j, 0} \stackrel{\text { def }}{=}$ $\psi_{j}$ and its shadows $\psi_{h, j, f}$ so that same relations as (21) are satisfied

$$
\sum_{\ell=0}^{2} \sum_{i=0}^{2} M_{\ell, i}\left(-\alpha_{j}+\lambda-\ell+\mu-i\right) \psi_{\lambda-\ell, j, \mu-i}(\varphi)=0, \quad \forall \lambda, \mu \geq 0
$$

Explicit instances of equation (22) are provided in sections 4 and 5. Formulas for the functions $\psi_{\ell, k, i}(\varphi)$ are given in [12] in case of homogeneous Neumann BCs.

Quasidual functions (QDF) are defined as the sum of a dual eigenfunction and a finite number of its dual shadows. To each QDF is associated an exponent $\alpha_{j}$, two non-negative cut-off integers $n$ and $m$ and a (test) function $B_{j}=B_{j}(\theta)$, defining the function $K_{n, m}^{\left(\alpha_{j}\right)}\left[B_{j}\right]$ as:

$$
K_{n, m}^{\left(\alpha_{j}\right)}\left[B_{j}\right] \stackrel{\text { def }}{=} \sum_{h=0,2,4, \cdots}^{m} \partial_{\theta}^{h} B_{j}(\theta) \rho^{-\alpha_{j}} \sum_{f=0}^{n}\left(\frac{\rho}{R}\right)^{h+f} \psi_{h, j, f}(\varphi)
$$

The angular functions $B_{j}$ are not specified at this stage and will be later chosen as trigonometric functions. When no confusion is possible, the mention of $B_{j}$ will be omitted in the notation of the QDF.

Multiplying $\Delta \tau=0$ by $K_{n, m}^{\left(\alpha_{j}\right)}$, then integrating over the subdomain $\Omega^{*}$ (see Figure 2) and applying Green's theorem one obtains,

$$
0=\int_{\partial \Omega^{*}}\left(K_{n, m}^{\left(\alpha_{j}\right)} \nabla \tau-\tau \nabla K_{n, m}^{\left(\alpha_{j}\right)}\right) \cdot \hat{n} d \Gamma+\int_{\Omega^{*}} \tau \Delta K_{n, m}^{\left(\alpha_{j}\right)} d \Omega
$$

The Laplace operator applied on $K_{n, m}^{\left(\alpha_{j}\right)}$, i.e. $\Delta K_{n, m}^{\left(\alpha_{j}\right)}$ is zero only when $n, m \rightarrow \infty$. For a finite $n$ and $m$ $\Delta K_{n, m}^{\left(\alpha_{j}\right)} \neq 0$, thus the last term in (24) does not vanish in general. 
On the two flat surfaces $\Gamma_{1}$ and $\Gamma_{2}$ homogeneous boundary conditions are prescribed thus either $\tau=0$ and $K_{n, m}^{\left(\alpha_{j}\right)}=0$, or $\partial_{\hat{n}} \tau=0$ and $\partial_{\hat{n}} K_{n, m}^{\left(\alpha_{j}\right)}=0$, for a circular closed edge thus (24) reduces to:

$$
\begin{aligned}
0 & =\left.\int_{0}^{2 \pi} \int_{\varphi_{1}}^{\varphi_{2}}\left(K_{n, m}^{\left(\alpha_{j}\right)} \nabla \tau-\tau \nabla K_{n, m}^{\left(\alpha_{j}\right)}\right) \cdot \hat{n}\right|_{\rho_{0}}\left(R+\rho_{0} \cos \varphi\right) \rho_{0} d \varphi d \theta \\
& +\left.\int_{0}^{2 \pi} \int_{\varphi_{2}}^{\varphi_{1}}\left(K_{n, m}^{\left(\alpha_{j}\right)} \nabla \tau-\tau \nabla K_{n, m}^{\left(\alpha_{j}\right)}\right) \cdot \hat{n}\right|_{\rho_{1}}\left(R+\rho_{1} \cos \varphi\right) \rho_{1} d \varphi d \theta+\int_{\Omega^{*}} \tau \Delta K_{n, m}^{\left(\alpha_{j}\right)} d \Omega .
\end{aligned}
$$

Since $\left.\nabla \cdot \hat{n}\right|_{\rho_{0}}=\partial_{\rho}$ and $\left.\nabla \cdot \hat{n}\right|_{\rho_{1}}=-\partial_{\rho}$, we obtain:

$$
\begin{aligned}
& \int_{0}^{2 \pi} \int_{\varphi_{1}}^{\varphi_{2}}\left(K_{n, m}^{\left(\alpha_{j}\right)} \partial_{\rho} \tau-\tau \partial_{\rho} K_{n, m}^{\left(\alpha_{j}\right)}\right)_{\rho_{0}}\left(R+\rho_{0} \cos \varphi\right) \rho_{0} d \varphi d \theta \\
& =\int_{0}^{2 \pi} \int_{\varphi_{1}}^{\varphi_{2}}\left(K_{n, m}^{\left(\alpha_{j}\right)} \partial_{\rho} \tau-\tau \partial_{\rho} K_{n, m}^{\left(\alpha_{j}\right)}\right)_{\rho_{1}}\left(R+\rho_{1} \cos \varphi\right) \rho_{1} d \varphi d \theta-\int_{\Omega^{*}} \tau \Delta K_{n, m}^{\left(\alpha_{j}\right)} d \Omega .
\end{aligned}
$$

Definition 2. For $\rho>0$ small enough, we define the surface-integral $J_{\rho}\left[\tau, K_{n, m}^{\left(\alpha_{j}\right)}\right]$ over the torus of minor radius $\rho$ and major radius $R$ that surrounds the circular edge by:

$$
J_{\rho}\left[\tau, K_{n, m}^{\left(\alpha_{j}\right)}\right] \stackrel{\text { def }}{=} \int_{0}^{2 \pi} \int_{\varphi_{1}}^{\varphi_{2}}\left(K_{n, m}^{\left(\alpha_{j}\right)} \partial_{\rho} \tau-\tau \partial_{\rho} K_{n, m}^{\left(\alpha_{j}\right)}\right)_{\rho}(R+\rho \cos \varphi) \rho d \varphi d \theta
$$

With the new notation, (26) becomes

$$
J_{\rho_{0}}\left[\tau, K_{n, m}^{\left(\alpha_{j}\right)}\right]-J_{\rho_{1}}\left[\tau, K_{n, m}^{\left(\alpha_{j}\right)}\right]=-\int_{\Omega^{*}} \tau \Delta K_{n, m}^{\left(\alpha_{j}\right)} d \Omega
$$

In contrast with the homogeneous 2-D case as shown in section $2, \operatorname{cf}(7)$, the integral $J_{\rho}\left[\tau, K_{n, m}^{\left(\alpha_{j}\right)}\right]$ is surfacedependent, i.e., it depends on $\rho$ :

Lemma 3. For any chosen $\tau$ satisfying $\Delta \tau=0$ and zero BC's in a neighborhood of the circular edge, for any chosen positive integers $j, n$ and $m$, for any smooth chosen function $B_{j}$, there holds for $\rho_{0} \geq \rho_{1}>0$ small enough

$$
J_{\rho_{0}}\left[\tau, K_{n, m}^{\left(\alpha_{j}\right)}\right]-J_{\rho_{1}}\left[\tau, K_{n, m}^{\left(\alpha_{j}\right)}\right]=\underset{\rho_{0} \rightarrow 0}{\mathcal{O}}\left(\left.\rho^{\alpha_{1}-\alpha_{j}+\min \{m, n\}+1}\right|_{\rho_{1}} ^{\rho_{0}}\right)
$$

Proof. We have to evaluate $\Delta K_{n, m}^{\left(\alpha_{j}\right)}$. For this we consider $\widetilde{\Delta} K_{n, m}^{\left(\alpha_{j}\right)}$. Applying (16), and in similarity with (19) we find

$$
\begin{aligned}
& \widetilde{\Delta} K_{n, m}^{\left(\alpha_{j}\right)}= \rho^{-\alpha_{j}} \sum_{\ell=0}^{2} \sum_{i=0}^{2} \sum_{h=0}^{m} \sum_{f=0}^{n}\left(\frac{\rho}{R}\right)^{i+\ell+f+h} \partial_{\theta}^{\ell+h} B_{j} M_{\ell, i}\left(-\alpha_{j}+h+f\right) \psi_{h, j, f} \\
&=\rho^{-\alpha_{j}} \sum_{\lambda=0}^{m+2} \sum_{\mu=0}^{n+2} \sum_{\ell=0}^{\min \{2, m+2-\lambda\}} \sum_{i=0}^{\min \{2, n+2-\mu\}}\left(\frac{\rho}{R}\right)^{\lambda+\mu} \partial_{\theta}^{\lambda} B_{j} \\
& M_{\ell, i}\left(-\alpha_{j}+\lambda-\ell+\mu-i\right) \psi_{\lambda-\ell, j, \mu-i} .
\end{aligned}
$$


Using (22) we observe that the terms involving values of $\lambda$ and $\mu$ such that $\min \{2, m+2-\lambda\}=2$ and $\min \{2, n+2-\mu\}=2$ cancel in the sum (31), so it becomes

$$
\begin{aligned}
\widetilde{\Delta} K_{n, m}^{\left(\alpha_{j}\right)} & =\rho^{-\alpha_{j}} \sum_{\lambda=m+1}^{m+2} \sum_{\ell=0}^{m+2-\lambda} \sum_{\mu=0}^{n+2} \sum_{i=0}^{\min \{2, n+2-\mu\}}\left(\frac{\rho}{R}\right)^{\lambda+\mu} \partial_{\theta}^{\lambda} B_{j} \\
& +\rho^{-\alpha_{j}} \sum_{\lambda=0}^{m} \sum_{\ell=0}^{2} \sum_{\mu=n+1}^{n+2} \sum_{i=0}^{n+2-\mu}\left(\frac{\rho}{R}\right)^{\lambda+\mu} \partial_{\theta}^{\lambda} B_{j} \\
M_{\ell, i}\left(-\alpha_{j}+\lambda-\ell+\mu-i\right) \psi_{\lambda-\ell, j, \mu-i} & \ell+\mu-i) \psi_{\lambda-\ell, j, \mu-i} .
\end{aligned}
$$

Then formula (29) is a straightforward consequence of the expansion (15) of $\tau$, and of the identities (9), (32) and (28).

3.4. Extracting circular GEFIFs using the quasidual-function method. We now prove the following theorem that shows how the $J_{\rho}$ integral evaluated with the quasi-dual functions (23) allow an accurate evaluation of moments of the coefficients $A_{k}$ in the expansion (15).

Theorem 4. Let the function $\tau$ satisfy interior equation (10) with boundary conditions (11) or (12). With $K_{n, m}^{\left(\alpha_{j}\right)}\left[B_{j}\right]$ defined by (23) and $D_{j}$ in (7), there hold the following formulas for the extraction of the GEFIF $A_{j}$ of $\tau, c f(15)$ :

(i) Concerning the first GEFIF $A_{1}$, we have:

$$
J_{\rho}\left[\tau, K_{n, m}^{\left(\alpha_{1}\right)}\left[B_{1}\right]\right]=2 \alpha_{1} R D_{1}\left(\int_{0}^{2 \pi} A_{1} B_{1} d \theta\right)+\mathcal{O}\left(\rho^{1+\min \{n, m\}}\right)
$$

(ii) This formula generalizes to the next GEFIF $A_{j}, j=2,3, \ldots$ if there are no resonances (i.e. in the case when $\alpha_{j}-\alpha_{k}$ is not an integer for $k<j$ ):

$$
J_{\rho}\left[\tau, K_{n, m}^{\left(\alpha_{j}\right)}\left[B_{j}\right]\right]=2 \alpha_{j} R D_{j}\left(\int_{0}^{2 \pi} A_{j} B_{j} d \theta\right)+\mathcal{O}\left(\rho^{\alpha_{1}-\alpha_{j}+1+\min \{n, m\}}\right)
$$

Returning to the definitions (27) and (7), formula (34) results in:

$$
\begin{aligned}
\int_{0}^{2 \pi} & \int_{\varphi_{1}}^{\varphi_{2}}\left(K_{n, m}^{\left(\alpha_{j}\right)}\left[B_{j}\right] \partial_{\rho} \tau-\tau \partial_{\rho} K_{n, m}^{\left(\alpha_{j}\right)}\left[B_{j}\right]\right)_{\rho}(R+\rho \cos \varphi) \rho d \varphi d \theta \\
= & \left(\int_{0}^{2 \pi} A_{j} B_{j} d \theta\right)\left(2 \alpha_{j} R \int_{\varphi_{1}}^{\varphi_{2}} \phi_{j}^{2} d \varphi\right)+\mathcal{O}\left(\rho^{\alpha_{1}-\alpha_{j}+1+\min \{n, m\}}\right) .
\end{aligned}
$$

This means that the $J_{\rho}$ integral used with the QDF $K_{n, m}^{\left(\alpha_{j}\right)}\left[B_{j}\right]$ allows the extraction of the moment of $A_{j}$ against $B_{j}$ if $n$ and $m$ are chosen larger than $\alpha_{j}-\alpha_{1}$. Then the error $\mathcal{O}\left(\rho^{\alpha_{1}-\alpha_{j}+1+\min \{n, m\}}\right)$ converges to 0 as $\rho \rightarrow 0$. In practice, the use of larger values for $n$ and $m$ allows to take larger values of $\rho$ for a same level of error. This permits to combine this extraction method with not strongly refined meshes.

Remark 5. In the axisymmetric case, all functions $A_{k}$ are constant along the edge. Thus we choose $B_{j}$ as constant functions. This corresponds to the angular mode of order 0 . Hence the associated QDFs satisfy $K_{n, 0}^{\left(\alpha_{j}\right)}\left[B_{j}\right]=K_{n, m}^{\left(\alpha_{j}\right)}\left[B_{j}\right]$ for all $m$.

Remark 6. In the general case, taking $m=n$ optimizes the number of shadow functions with respect to $m$ and $n$ so that (34) becomes

$$
J_{\rho}\left[\tau, K_{n, m}^{\left(\alpha_{j}\right)}\left[B_{j}\right]\right]=2 \alpha_{j} R D_{j}\left(\int_{0}^{2 \pi} A_{j} B_{j} d \theta\right)+\mathcal{O}\left(\rho^{\alpha_{1}-\alpha_{j}+1+n}\right)
$$

In fact, when $m$ is even we have $K_{n, m}^{\left(\alpha_{j}\right)}=K_{n, m+1}^{\left(\alpha_{j}\right)}$, thus $n=m+1$ is optimal. 
Remark 7. The generalization of this method to the case of the equation $\Delta \tau=F$ with a smooth function $F$ depends on the values of $F$ along the edge. If $F$ is zero with its derivatives, the method can be used without modification. In the opposite case, one has first to determine a particular solution of the equation $\Delta \tau^{\text {pol }}=F^{\text {pol }}$ with BCs, where $F^{\mathrm{pol}}$ the Taylor expansion of $F$ along the edge. In a second step, the method will be used for $\tau-\tau^{\text {pol }}$ and provide the GEFIF of $\tau$.

Proof. of Theorem 4. First we investigate the surface-integral $J_{\rho}\left[\tau, K_{n, m}^{\left(\alpha_{j}\right)}\right]$ when $\tau$ is replaced by finite sums of primal functions with their shadows. Recall definition (23)

$$
K_{n, m}^{\left(\alpha_{j}\right)}\left[B_{j}\right]=\sum_{h=0,2,4, \cdots}^{m} \partial_{\theta}^{h} B_{j}(\theta) \rho^{-\alpha_{j}} \sum_{f=0}^{n}\left(\frac{\rho}{R}\right)^{h+f} \psi_{h, j, f}(\varphi) .
$$

Likewise we define finite singular expansions

$$
\tau_{n, m}^{\left(\alpha_{k}\right)}\left[A_{k}\right] \stackrel{\text { def }}{=} \sum_{\ell=0,2,4, \cdots}^{m} \partial_{\theta}^{\ell} A_{k}(\theta) \rho^{\alpha_{k}} \sum_{i=0}^{n}\left(\frac{\rho}{R}\right)^{\ell+i} \phi_{\ell, k, i}(\varphi) .
$$

Although in the expansion (15) the series associated with the eigenvalue $\alpha_{k}$ includes an infinite number of terms, for the mathematical analysis we first consider a finite sum. We investigate the surface integrals $J_{\rho}\left[\tau_{n^{\prime}, m^{\prime}}^{\left(\alpha_{k}\right)}\left[A_{k}\right], K_{n, m}^{\left(\alpha_{j}\right)}\left[B_{j}\right]\right]$. Instead of (28), we have

$$
\begin{aligned}
J_{\rho_{0}}\left[\tau_{n^{\prime}, m^{\prime}}^{\left(\alpha_{k}\right)}\left[A_{k}\right], K_{n, m}^{\left(\alpha_{j}\right)}\left[B_{j}\right]\right]-J_{\rho_{1}}\left[\tau_{n^{\prime}, m^{\prime}}^{\left(\alpha_{k}\right)}\left[A_{k}\right], K_{n, m}^{\left(\alpha_{j}\right)}\left[B_{j}\right]\right] \\
=\int_{\Omega^{*}} K_{n, m}^{\left(\alpha_{j}\right)}\left[B_{j}\right] \Delta \tau_{n^{\prime}, m^{\prime}}^{\left(\alpha_{k}\right)}\left[A_{k}\right]-\tau_{n^{\prime}, m^{\prime}}^{\left(\alpha_{k}\right)}\left[A_{k}\right] \Delta K_{n, m}^{\left(\alpha_{j}\right)}\left[B_{j}\right] d \Omega .
\end{aligned}
$$

Since $\widetilde{\Delta} \tau_{n^{\prime}, m^{\prime}}^{\left(\alpha_{k}\right)}\left[A_{k}\right]$ satisfies mutatis mutandis (32), one obtains that

$$
K_{n, m}^{\left(\alpha_{j}\right)}\left[B_{j}\right] \widetilde{\Delta} \tau_{n^{\prime}, m^{\prime}}^{\left(\alpha_{k}\right)}\left[A_{k}\right]-\tau_{n^{\prime}, m^{\prime}}^{\left(\alpha_{k}\right)}\left[A_{k}\right] \widetilde{\Delta} K_{n, m}^{\left(\alpha_{j}\right)}\left[B_{j}\right]=\rho^{\alpha_{k}-\alpha_{j}} \sum_{\nu=1+\min \left\{n, m, n^{\prime}, m^{\prime}\right\}}^{n+m+n^{\prime}+m^{\prime}+4} \rho_{\nu}^{\nu} F_{\nu}(\varphi, \theta) .
$$

where the functions $F_{\nu}$ do not depend on $\rho$, but depend on all other data. Taking the relation (9) between $\Delta$ and $\widetilde{\Delta}$ into account and the fact that $d \Omega=\rho(R+\rho \cos \varphi) d \rho d \varphi d \theta$ we find a sequence of real coefficients $G_{\nu}$ such that

$$
\begin{aligned}
\int_{\Omega^{*}} K_{n, m}^{\left(\alpha_{j}\right)}\left[B_{j}\right] \Delta \tau_{n^{\prime}, m^{\prime}}^{\left(\alpha_{k}\right)}\left[A_{k}\right]-\tau_{n^{\prime}, m^{\prime}}^{\left(\alpha_{k}\right)}\left[A_{k}\right] \Delta K_{n, m}^{\left(\alpha_{j}\right)}\left[B_{j}\right] d \Omega & \\
& =\sum_{\nu=1+\min \left\{n, m, n^{\prime}, m^{\prime}\right\}}^{\infty}\left(\left.\rho^{\alpha_{k}-\alpha_{j}+\nu}\right|_{\rho_{1}} ^{\rho_{0}}\right) G_{\nu}
\end{aligned}
$$

The series in the right hand side of (41) is convergent for $\rho_{0}$ small enough. We combine (39) with (41) for $\rho_{0}=\rho$ and $\rho_{1}=\varepsilon$ :

$$
\begin{aligned}
J_{\rho}\left[\tau_{n^{\prime}, m^{\prime}}^{\left(\alpha_{k}\right)}\left[A_{k}\right], K_{n, m}^{\left(\alpha_{j}\right)}\left[B_{j}\right]\right]- & J_{\varepsilon}\left[\tau_{n^{\prime}, m^{\prime}}^{\left(\alpha_{k}\right)}\left[A_{k}\right], K_{n, m}^{\left(\alpha_{j}\right)}\left[B_{j}\right]\right] \\
= & \sum_{\nu=1+\min \left\{n, m, n^{\prime}, m^{\prime}\right\}}^{\infty} \rho^{\alpha_{k}-\alpha_{j}+\nu} G_{\nu}-\sum_{\nu=1+\min \left\{n, m, n^{\prime}, m^{\prime}\right\}}^{\infty} \varepsilon^{\alpha_{k}-\alpha_{j}+\nu} G_{\nu} .
\end{aligned}
$$

We consider $\rho$ as fixed and let $\varepsilon$ tend to 0 . We can write

$$
J_{\varepsilon}\left[\tau_{n^{\prime}, m^{\prime}}^{\left(\alpha_{k}\right)}\left[A_{k}\right], K_{n, m}^{\left(\alpha_{j}\right)}\left[B_{j}\right]\right]=C+\sum_{\nu=1+\min \left\{n, m, n^{\prime}, m^{\prime}\right\}}^{\infty} \varepsilon^{\alpha_{k}-\alpha_{j}+\nu} G_{\nu} .
$$

where $C$ is a constant independent of $\varepsilon$. 
Now, using (27) and expressions (37)-(38), we find coefficients $H_{\nu}^{k, j \mid n, m, n^{\prime}, m^{\prime}}\left[A_{k}, B_{j}\right]$ given by

$$
\begin{aligned}
& H_{\nu}^{k, j \mid n, m, n^{\prime}, m^{\prime}}\left[A_{k}, B_{j}\right]= \\
& R^{1-\nu} \underbrace{\sum_{h=0,2, f=0}^{m} \sum_{\ell=0,2, i=0}^{n} \sum_{m^{\prime}}^{n^{\prime}}}_{h+f+\ell+i=\nu}\left(\int_{0}^{2 \pi} \partial_{\theta}^{\ell} A_{k}(\theta) \partial_{\theta}^{h} B_{j}(\theta) d \theta\right)\left(\alpha_{k}+\alpha_{j}+\ell+i-h-f\right) \int_{\varphi_{1}}^{\varphi_{2}} \phi_{\ell, k, i}(\varphi) \psi_{h, j, f}(\varphi) d \varphi \\
& +R^{1-\nu} \underbrace{\sum_{h=0,2, f=0}^{m} \sum_{\ell=0,2, i=0}^{n} \sum_{\ell=0}^{m^{\prime}}}_{h+f+\ell+i=\nu-1}\left(\int_{0}^{n^{\prime}} \partial_{\theta}^{\ell \pi} A_{k}(\theta) \partial_{\theta}^{h} B_{j}(\theta) d \theta\right)\left(\alpha_{k}+\alpha_{j}+\ell+i-h-f\right) \int_{\varphi_{1}}^{\varphi_{2}} \phi_{\ell, k, i}(\varphi) \psi_{h, j, f}(\varphi) \cos \varphi d \varphi
\end{aligned}
$$

such that for any $\rho$ small enough

$$
J_{\rho}\left[\tau_{n^{\prime}, m^{\prime}}^{\left(\alpha_{k}\right)}\left[A_{k}\right], K_{n, m}^{\left(\alpha_{j}\right)}\left[B_{j}\right]\right]=\sum_{\nu=0}^{n+m+n^{\prime}+m^{\prime}+1} \rho^{\alpha_{k}-\alpha_{j}+\nu} H_{\nu}^{k, j \mid n, m, n^{\prime}, m^{\prime}}\left[A_{k}, B_{j}\right] .
$$

Using this for $\rho=\varepsilon$ and combining with (43) we obtain

$$
\sum_{\nu=0}^{n+m+n^{\prime}+m^{\prime}+1} \varepsilon^{\alpha_{k}-\alpha_{j}+\nu} H_{\nu}^{k, j \mid n, m, n^{\prime}, m^{\prime}}\left[A_{k}, B_{j}\right]=C+\sum_{\nu=1+\min \left\{n, m, n^{\prime}, m^{\prime}\right\}}^{\infty} \varepsilon^{\alpha_{k}-\alpha_{j}+\nu} G_{\nu} .
$$

We note that by definition, the coefficient $H_{\nu}^{k, j \mid n, m, n^{\prime}, m^{\prime}}\left[A_{k}, B_{j}\right]$ does not depend on $n, m, n^{\prime}, m^{\prime}$ as soon as $\nu \leq \min \left\{n, m, n^{\prime}, m^{\prime}\right\}$. We denote it by $H_{\nu}^{k, j}\left[A_{k}, B_{j}\right]$.

Thus, identifying the powers of $\varepsilon$ in (46), we find that

$$
H_{\nu}^{k, j}\left[A_{k}, B_{j}\right]=0, \quad \forall \nu \leq \min \left\{n, m, n^{\prime}, m^{\prime}\right\} \text { such that } \alpha_{k}-\alpha_{j}+\nu \neq 0 .
$$

In contrast, when $\alpha_{k}-\alpha_{j}+\nu=0$, the factor $H_{\nu}^{k, j}\left[A_{k}, B_{j}\right]$ does not need to be zero. Typically $\alpha_{k}-\alpha_{j}+\nu=0$ for $k=j$ and $\nu=0$. In this case, (44) yields

$$
\begin{aligned}
& \text { If } k=j, \quad H_{0}^{k, j}\left[A_{k}, B_{j}\right] \equiv H_{0}^{k, k}\left[A_{k}, B_{k}\right]= \\
& \qquad\left(\int_{0}^{2 \pi} A_{k}(\theta) B_{k}(\theta) d \theta\right)\left(2 \alpha_{k} R \int_{\varphi_{1}}^{\varphi_{2}} \phi_{k}^{2}(\varphi) d \varphi\right) .
\end{aligned}
$$

From (45) and (47) we deduce for $m^{\prime} \geq m$ and $n^{\prime} \geq n$

$$
J_{\rho}\left[\tau_{n^{\prime}, m^{\prime}}^{\left(\alpha_{k}\right)}\left[A_{k}\right], K_{n, m}^{\left(\alpha_{j}\right)}\left[B_{j}\right]\right]=\left\{\begin{array}{r}
\underset{\mathcal{O}}{\mathcal{O}}\left(\rho^{\alpha_{k}-\alpha_{j}+1+\min \{n, m\}}\right) \\
\text { if } \alpha_{j}-\alpha_{k} \notin\{0, \ldots, \min \{n, m\}\} \\
H_{\nu}^{k, j}\left[A_{k}, B_{j}\right]+\underset{\rho \rightarrow 0}{\mathcal{O}}\left(\rho^{\alpha_{k}-\alpha_{j}+1+\min \{n, m\}}\right) \\
\text { if } \alpha_{j}-\alpha_{k}:=\nu \in\{0, \ldots, \min \{n, m\}\}
\end{array}\right.
$$

By linearity we deduce from (15) and (49) (considering the entire solution $\tau$, so that we can take $n^{\prime} \geq n$ and $m^{\prime} \geq m$ )

$$
\begin{aligned}
J_{\rho}\left[\tau, K_{n, m}^{\left(\alpha_{j}\right)}\left[B_{j}\right]\right] & = \\
& \sum_{k} \rho^{\alpha_{k}-\alpha_{j}}\left\{\sum_{\nu=0}^{\min \{n, m\}} \rho^{\nu} H_{\nu}^{k, j}\left[A_{k}, B_{j}\right]+{\underset{\mathcal{O}}{\rho \rightarrow 0}}\left(\rho^{1+\min \{n, m\}}\right)\right\}
\end{aligned}
$$


In particular, if $j=1$ the only non-zero coefficient $H_{\nu}^{k, j}$ is for $k=j=1$ and $\nu=0$. Combining (47)-(50), we have proved the theorem.

In the following sections, we specify the use of the extraction formula (35) for axisymmetric as well as nonaxisymmetric solutions in domains with circular edges. We also extend the method to cases for which $\alpha_{k}-\alpha_{j}$ are integers ("resonant" case). Numerical tests will demonstrate the efficiency of (35) for extracting GEFIFs from numerical solutions.

\section{Extracting Circular GEFIFs For AxisymmetriC SOlutions by THE QDFM}

In the axisymmetric case the coefficients $A_{k}$ are $\theta$ independent, so the solution $\tau$ may be expressed as follows

$$
\tau(\rho, \varphi)=\sum_{k \geq 0} A_{k} \rho^{\alpha_{k}} \sum_{i \geq 0}\left(\frac{\rho}{R}\right)^{i} \phi_{0, k, i}(\varphi)
$$

Thus, for the QDFs $K_{n, 0}^{\left(\alpha_{j}\right)}\left[B_{j}\right]$ in the axisymmetric case, we take $B_{j}$ being $\theta$ independent (a constant) and $m=0$ :

$$
K_{n, 0}^{\left(\alpha_{j}\right)}(\rho, \varphi)\left[B_{j}\right]=B_{j} \rho^{-\alpha_{j}} \sum_{f=0}^{n}\left(\frac{\rho}{R}\right)^{f} \psi_{0, j, f}(\varphi) .
$$

The explicit equations for the dual eigenfunctions and their shadows $\psi_{0, j, f}(\varphi)$ are obtained from (22) and [15]:

$$
\begin{aligned}
\alpha_{j}^{2} \psi_{0, j, 0}+\psi_{0, j, 0}^{\prime \prime}= & 0, \\
\left(-\alpha_{j}+1\right)^{2} \psi_{0, j, 1}+\psi_{0, j, 1}^{\prime \prime}= & -\left(-\alpha_{j} \cos \varphi \psi_{0, j, 0}-\sin \varphi \psi_{0, j, 0}^{\prime}\right), \\
\left(-\alpha_{j}+i\right)^{2} \psi_{0, j, f}+\psi_{0, j, f}^{\prime \prime}= & -\left[\left(-\alpha_{j}+f\right)\left(-\alpha_{j}+f-1\right) \cos \varphi \psi_{0, j, f-1}\right. \\
& \left.-\sin \varphi \psi_{0, j, f-1}^{\prime}+\cos \varphi \psi_{0, j, f-1}^{\prime \prime}\right], \quad f \geq 2,
\end{aligned}
$$

for $\varphi_{1}<\varphi<\varphi_{2}$, completed by homogeneous BCs

$$
\begin{aligned}
\psi_{0, j, f} & =0 \text { on } \varphi_{1}, \varphi_{2} \text { homogeneous Drichlet BCs } \\
\partial_{\varphi} \psi_{0, j, f} & =0 \text { on } \varphi_{1}, \varphi_{2} \text { homogeneous Neumann BCs. }
\end{aligned}
$$

Since $B_{j}$ is constant, the QDF $K_{n, 0}^{\left(\alpha_{j}\right)}\left[B_{j}\right]$ coincides with $K_{n, n}^{\left(\alpha_{j}\right)}\left[B_{j}\right]$. Taking into account that $\int_{0}^{2 \pi} A_{j} B_{j} d \theta=$ $2 \pi A_{j} B_{j}$, we find that formula (34) becomes in the axisymmetric case:

$$
J_{\rho}\left[\tau, K_{n, 0}^{\left(\alpha_{j}\right)}\left[B_{j}\right]\right]=4 \pi \alpha_{j} R D_{j} A_{j} B_{j}+\mathcal{O}\left(\rho^{\alpha_{1}-\alpha_{j}+n+1}\right),
$$

Therefore, choosing $B_{j}$ as:

$$
B_{j}=\frac{1}{4 \pi \alpha_{j} R D_{j}}
$$

equations (58) and (60) result in $A_{j}$ alone with a remainder dependent on $\rho$.

We illustrate this formula by several particular examples. We even find an improvement for a circular crack with Neumann BC's: For a certain finite set of integers $j$ and $n$ we have found that, with $B_{j}$ given by (59)

$$
J_{\rho}\left[\tau, K_{n, 0}^{\left(\alpha_{j}\right)}\left[B_{j}\right]\right]=A_{j}+\mathcal{O}\left(\rho^{2\left(\alpha_{1}-\alpha_{j}+n+1\right)}\right) .
$$

Remark 8. In the general situation of non-axisymmetric data, the use of constant $B_{j}$ given by (59) provides an approximation of the mean value of the GESIF

$$
\frac{1}{2 \pi} \int_{0}^{2 \pi} A_{j}(\theta) d \theta
$$

with the same rates (58) or (60). 
4.1. A circular crack with homogenous Neumann BCs - Axisymmetric case. For an axisymmetric solution of a circular crack with homogenous Neumann BCs, $\omega=2 \pi,\left(\varphi_{1}=-\pi, \varphi_{2}=\pi\right)$ the eigenvalues are $\alpha_{k}=0, \frac{1}{2}, 1, \frac{3}{2}, 2, \frac{5}{2}, 3, \cdots$.

We represent $\tau$ up to $\mathcal{O}\left(\rho^{11}\right)$ as follows (the explicit expressions for $\phi_{0, k, i}$ are provided in [12]):

$$
\begin{aligned}
\tau & =A_{0}+A_{1} \rho^{1 / 2} \sum_{i=0}^{10}\left(\frac{\rho}{R}\right)^{i} \phi_{0,1, i}+A_{2} \rho \sum_{i=0}^{10}\left(\frac{\rho}{R}\right)^{i} \phi_{0,2, i} \\
& +A_{3} \rho^{3 / 2} \sum_{i=0}^{9}\left(\frac{\rho}{R}\right)^{i} \phi_{0,3, i}+A_{4} \rho^{2} \sum_{i=0}^{9}\left(\frac{\rho}{R}\right)^{i} \phi_{0,4, i}+ \\
& +\cdots+A_{21} \rho^{21 / 2} \phi_{0,21,0}+\mathcal{O}\left(\rho^{11}\right) .
\end{aligned}
$$

Remark 9. Knowing the explicit expansion (61) is not required to implement the QDFM. We provide it for two reasons: First to investigate the cancelations leading to the super convergence rate (60), and second to implement boundary data in numerical models, so that the exact solution is close to (61).

Remark 10. The eigenfunctions and shadows associated with the integer eigenvalues are orthogonal to the dual eigenfunctions and dual shadows associated with half-integer eigenvalues under the $J_{\rho}$ integral. This is the reason that the terms associated with $A_{0}, A_{2}, A_{4}, \cdots$ are absent from formulas (66) and sequel.

Because (61) is independent of $\theta$, the first terms $\phi_{0, j, 0}=\phi_{j}$ and $\psi_{0, j, 0}=\psi_{j}$ of the primal and dual singular functions are given by

$$
\phi_{j}(\varphi)=\psi_{j}(\varphi)=\left\{\begin{array}{ll}
\cos \frac{j \varphi}{2} & j=0,2,4, \cdots \\
\sin \frac{j \varphi}{2} & j=1,3,5, \cdots
\end{array} \quad \varphi \in(-\pi, \pi) .\right.
$$

Thus according to (7) $D_{j}=\pi$ and we choose as in (59)

$$
B_{j}=\frac{1}{4 \pi^{2} \alpha_{j} R}=\frac{1}{2 j \pi^{2} R} .
$$

4.1.1. Extracting $A_{1}$. To extract $A_{1}$, according to (63) we take $B_{1}=1 /\left(2 \pi^{2} R\right)$. In order to simplify notations, we write

$$
K_{n, 0}^{(1 / 2)} \stackrel{\text { def }}{=} K_{n, 0}^{(1 / 2)}\left[B_{1}\right] \quad \text { with } \quad B_{1}=\frac{1}{2 \pi^{2} R} .
$$

For a crack the quasi-dual functions $K_{n, 0}^{(1 / 2)}$ have a particular simple form (when compared to other ones, see [12]). We find

$$
K_{n, 0}^{(1 / 2)}=\frac{1}{2 \pi^{2} R} \rho^{-1 / 2}\left[\sum_{i=0}^{n}(-1)^{i} \beta_{i} \sin \frac{(2 i+1) \varphi}{2}\left(\frac{\rho}{R}\right)^{i}\right]
$$

with the constants $\beta_{i}$ given in Table 1 .

$$
\begin{array}{ccccccccccc}
\beta_{0} & \beta_{1} & \beta_{2} & \beta_{3} & \beta_{4} & \beta_{5} & \beta_{6} & \beta_{7} & \beta_{8} & \beta_{9} & \beta_{10} \\
1 & \frac{1}{4} & \frac{3}{32} & \frac{5}{128} & \frac{35}{2048} & \frac{63}{8192} & \frac{231}{65536} & \frac{429}{262144} & \frac{6435}{8388608} & \frac{12155}{33554432} & \frac{46189}{268435456} \\
\multicolumn{1}{c|}{\text { TABLE }} & \text { 1. Coefficients in the expansion (65) of the QDF } K_{n, 0}^{(1 / 2)} .
\end{array}
$$

One may extrapolate from the values in Table 1 an explicit expression for $K_{\infty, 0}^{(1 / 2)}$ as shown in Appendix A.

We extract $A_{1}$ by (35) using $K_{0,0}^{(1 / 2)}, \ldots, K_{3,0}^{(1 / 2)}$. The expected convergence rates as $\rho \rightarrow 0$ are given in (49). Here, relying on the explicit expressions of the primal singular and shadow angular functions $\phi_{0, k, i}$ and 
their dual analogues $\psi_{0, j, f}$ provided in [12], we calculate $J_{\rho_{0}}\left[\tau, K_{n, 0}^{(1 / 2)}\right]$ for $n=0, \ldots, 3$ and obtain the following.

$$
\begin{aligned}
& J_{\rho_{0}}\left[\tau, K_{0,0}^{(1 / 2)}\right]=A_{1}\left(1-\frac{\rho_{0}^{3}}{64 R^{3}}+\cdots\right)+A_{3}\left(\frac{\rho_{0}^{2}}{4 R}+\cdots\right) \\
& +A_{5}\left(-\frac{\rho_{0}^{4}}{32 R^{2}}+\cdots\right)+A_{7}\left(\frac{\rho_{0}^{6}}{128 R^{3}}+\cdots\right)+\cdots=A_{1}+\mathcal{O}\left(\frac{\rho_{0}^{2}}{R}\right) \\
& J_{\rho_{0}}\left[\tau, K_{1,0}^{(1 / 2)}\right]=A_{1}\left(1-\frac{3 \rho_{0}^{5}}{2^{10} R^{5}}+\cdots\right)+A_{3}\left(\frac{3 \rho_{0}^{6}}{1120 R^{5}}+\cdots\right) \\
& +A_{5}\left(-\frac{3 \rho_{0}^{4}}{32 R^{2}}+\cdots\right)+A_{7}\left(\frac{\rho_{0}^{6}}{64 R^{3}}+\cdots\right)+A_{9}\left(-\frac{9 \rho_{0}^{8}}{2^{11} R^{4}}+\cdots\right) \\
& +\quad \cdots=A_{1}+\mathcal{O}\left(\frac{\rho_{0}^{4}}{R^{2}}\right) \\
& J_{\rho_{0}}\left[\tau, K_{2,0}^{(1 / 2)}\right]=A_{1}\left(1-\frac{75 \rho_{0}^{7}}{2^{17} R^{7}}+\cdots\right)+A_{3}\left(\frac{\rho_{0}^{8}}{2^{8} 7 R^{7}}+\cdots\right) \\
& +A_{5}\left(-\frac{5 \rho_{0}^{8}}{2^{9} 7 R^{6}}+\cdots\right)+A_{7}\left(\frac{5 \rho_{0}^{6}}{128 R^{3}}+\cdots\right)+A_{9}\left(-\frac{15 \rho_{0}^{8}}{2^{11} R^{4}}+\cdots\right) \\
& +\cdots=A_{1}+\mathcal{O}\left(\frac{\rho_{0}^{6}}{R^{3}}\right) \\
& J_{\rho_{0}}\left[\tau, K_{3,0}^{(1 / 2)}\right]=A_{1}\left(1-\frac{245 \rho_{0}^{9}}{2^{21} R^{9}}+\cdots\right)+A_{3}\left(\frac{\rho_{0}^{10}}{2^{8} 33 R^{9}}+\cdots\right) \\
& +A_{5}\left(-\frac{5 \rho_{0}^{10}}{2^{9} 33 R^{8}}+\cdots\right)+A_{7}\left(\frac{35 \rho_{0}^{10}}{2^{9} 99 R^{7}}+\cdots\right)+A_{9}\left(-\frac{35 \rho_{0}^{8}}{2^{11} R^{4}}+\cdots\right) \\
& +\cdots=A_{1}+\mathcal{O}\left(\frac{\rho_{0}^{8}}{R^{4}}\right)
\end{aligned}
$$

One may observe from formulas (66)-(69) that for a circular crack in an axisymmetric case, the first GEFIF $A_{1}$ may be extracted by the QDFM, using $K_{n, 0}^{(1 / 2)}$ with an improved remainder as in (60) when compared to (58):

$$
J_{\rho_{0}}\left[\tau, K_{n, 0}^{(1 / 2)}\right]=A_{1}+\mathcal{O}\left(\rho_{0}^{n+1}\left(\frac{\rho_{0}}{R}\right)^{n+1}\right)
$$

The remainder corresponds to the contribution of $A_{2 n+3}$ if it is non-zero. When compared with the proved generic rates of convergence, $\mathrm{cf}(49)$, which particularize as

$$
J_{\rho}\left[\tau_{n, 0}^{(k / 2)}\left[A_{k}\right], K_{n, 0}^{(1 / 2)}\right]= \begin{cases}A_{1}+\underset{\rho \rightarrow 0}{\mathcal{O}}\left(\rho^{1+n}\right) & \text { if } k=1, \\ \underset{\rho \rightarrow 0}{\mathcal{O}}\left(\rho^{(k-1) / 2+1+n}\right) & \text { if } k \geq 3,\end{cases}
$$

we observe that a coincidence of the rates occurs when $k=2 n+3$ and a practical improvement otherwise.

4.1.2. Extracting $A_{3}$ - the problem of resonance and its remedy. By same methods presented, we aim at computing $J_{\rho_{0}}\left[\tau, K_{n, 0}^{\left(\alpha_{3}=3 / 2\right)}\right]$ with

$$
K_{n, 0}^{(3 / 2)} \stackrel{\text { def }}{=} K_{n, 0}^{(3 / 2)}\left[B_{3}\right] \quad \text { with } \quad B_{3}=\frac{1}{6 \pi^{2} R},
$$


Since $\alpha_{3}>\alpha_{1}$, according to the general principles, $\mathrm{cf}$ (58), one should use a QDF which has at least one shadow, i.e., $K_{1,0}^{(3 / 2)}$ or one with a larger $n$. More precisely, the proved generic convergence rates, cf (49), particularize as

$$
J_{\rho}\left[\tau_{n, 0}^{(k / 2)}\left[A_{k}\right], K_{n, 0}^{(3 / 2)}\right]=\left\{\begin{array}{cl}
\underset{\rho \rightarrow 0}{\mathcal{O}}\left(\rho^{0}\right) & \text { if } k=1 \text { and } n=0, \\
\gamma A_{1}+\underset{\rho \rightarrow 0}{\mathcal{O}}\left(\rho^{n}\right) & \text { if } k=1 \text { and } n \geq 1, \\
A_{3}+\underset{\rho \rightarrow 0}{\mathcal{O}}\left(\rho^{1+n}\right) & \text { if } k=3, \\
\underset{\rho \rightarrow 0}{\mathcal{O}}\left(\rho^{(k-3) / 2+1+n}\right) & \text { if } k \geq 5,
\end{array}\right.
$$

where the constant $\gamma$ is a shorthand for the coefficient $H_{1}^{1,3}\left[A_{1}=1, B_{3}\right]$.

But since the remainders have de facto better decay properties, we may use $K_{0,0}^{(3 / 2)}$ as well. With

$$
K_{0,0}^{(3 / 2)}=\frac{1}{6 \pi^{2} R} \rho^{-3 / 2} \sin \frac{3 \varphi}{2},
$$

we find

$$
\begin{aligned}
J_{\rho_{0}}\left[\tau, K_{0,0}^{(3 / 2)}\right] & =A_{1}\left(\frac{1}{3 R}+\frac{5 \rho_{0}^{3}}{768 R^{4}}+\cdots\right)+A_{3}\left(1-\frac{3 \rho_{0}^{4}}{560 R^{4}}+\cdots\right) \\
& +A_{5}\left(\frac{\rho_{0}^{2}}{4 R}+\cdots\right)=A_{1} \frac{1}{3 R}+A_{3}+\mathcal{O}\left(\frac{\rho_{0}^{2}}{R}\right) .
\end{aligned}
$$

We encounter a "resonance" when trying to extract $A_{j}$ associated with $\alpha_{j}=\alpha_{k}+\nu$, so that $j>k$ and $\nu \in \mathbb{N}$. This "resonance" can be easily removed if we consider $K_{0,0}^{(1 / 2)}$, which extracts exactly $A_{1}$, see (66). Instead of $K_{0,0}^{(3 / 2)}$ only, we choose the extraction function:

$$
\breve{K}_{0,0}^{(3 / 2)} \stackrel{\text { def }}{=} K_{0,0}^{(3 / 2)}+c K_{0,0}^{(1 / 2)}
$$

with $c=-\frac{1}{3 R}$ so one obtains

$$
J_{\rho_{0}}\left[\tau, \breve{K}_{0,0}^{(3 / 2)}\right]=A_{3}+\mathcal{O}\left(\frac{\rho_{0}^{2}}{R}\right)
$$

which leads to the lowest order extraction formula for $A_{3}$.

If we try to use the QDF:

$$
K_{1,0}^{(3 / 2)}=\frac{1}{6 \pi^{2} R} \rho^{-3 / 2}\left[\sin \frac{3}{2} \varphi-\frac{1}{4} \sin \frac{5 \varphi}{2}\left(\frac{\rho}{R}\right)\right]
$$

then computing $J_{\rho_{0}}\left[\tau, K_{1,0}^{(3 / 2)}\right]$ with (61) results:

$$
\begin{aligned}
J_{\rho_{0}}\left[\tau, K_{1,0}^{(3 / 2)}\right] & =A_{1}\left(\frac{1}{3 R}+\frac{\rho_{0}^{3}}{192 R^{4}}+\cdots\right)+A_{3}\left(1-\frac{\rho_{0}^{2}}{12 R^{2}}+\cdots\right) \\
& +A_{5}\left(\frac{\rho_{0}^{4}}{96 R^{3}}+\cdots\right)+A_{7}\left(-\frac{9 \rho_{0}^{4}}{96 R^{2}}+\cdots\right)+\cdots \\
& =\frac{1}{3 R} A_{1}+A_{3}+\mathcal{O}\left(\frac{\rho_{0}^{2}}{R^{2}}\right)
\end{aligned}
$$

In view of (78) due to the resonance we construct again the modified QDF:

$$
\begin{aligned}
\breve{K}_{1,0}^{(3 / 2)} & \stackrel{\text { def }}{=} K_{1,0}^{(3 / 2)}-\frac{1}{3 R} K_{1,0}^{(1 / 2)} \\
& =\frac{1}{6 \pi^{2} R} \rho^{-3 / 2}\left[\sin \frac{3 \varphi}{2}-\frac{1}{4}\left(\frac{\rho}{R}\right) \sin \frac{5 \varphi}{2}-\left(\frac{\rho}{R}\right) \sin \frac{\varphi}{2}+\frac{1}{4}\left(\frac{\rho}{R}\right)^{2} \sin \frac{3 \varphi}{2}\right]
\end{aligned}
$$


This leads to the extraction formula for $A_{3}$ :

$$
J_{\rho_{0}}\left[\tau, \breve{K}_{1,0}^{(3 / 2)}\right]=A_{3}+\mathcal{O}\left(\frac{\rho_{0}^{2}}{R^{2}}\right)
$$

In a similar manner we may construct:

$$
\begin{aligned}
& \breve{K}_{2,0}^{(3 / 2) \stackrel{\text { def }}{=}} K_{2,0}^{(3 / 2)}-\frac{1}{3 R} K_{2,0}^{(1 / 2)} \\
&=\frac{1}{6 \pi^{2} R} \rho^{-3 / 2}[ {\left[\sin \frac{3 \varphi}{2}-\frac{1}{4}\left(\frac{\rho}{R}\right) \sin \frac{5 \varphi}{2}+\left(\frac{\rho}{R}\right)^{2}\left(\frac{1}{4} \sin \frac{3 \varphi}{2}+\frac{3}{32} \sin \frac{7 \varphi}{2}\right)\right.} \\
&\left.\quad-\left(\frac{\rho}{R}\right) \sin \frac{\varphi}{2}+\frac{1}{4}\left(\frac{\rho}{R}\right)^{2} \sin \frac{3 \varphi}{2}-\frac{3}{32}\left(\frac{\rho}{R}\right)^{3} \sin \frac{5 \varphi}{2}\right]
\end{aligned}
$$

and obtain that:

$$
J_{\rho_{0}}\left[\tau, \breve{K}_{2,0}^{(3 / 2)}\right]=A_{3}+\mathcal{O}\left(\frac{\rho_{0}^{4}}{R^{3}}\right)
$$

As one notices, the remedy to the "resonance" problem is to calculate $K_{n, 0}^{(3 / 2)}$ without taking the resonance with $\alpha_{1}$ into account. Then the modified QDF is defined

$$
\breve{K}_{n, 0}^{(3 / 2)} \stackrel{\text { def }}{=} K_{n, 0}^{(3 / 2)}-\frac{1}{3 R} K_{n, 0}^{(1 / 2)}
$$

that extracts $A_{3}$ for any $n$.

Remark 11. In the case of $A_{5}$, two resonances occur $\left(\alpha_{5}=\alpha_{1}+2=\alpha_{3}+1\right)$, then we construct the modified QDF by addition of the two QDFs associated with $\alpha_{1}$ and $\alpha_{3}$, multiplied by two constants:

$$
\breve{K}_{n, 0}^{(5 / 2)} \stackrel{\text { def }}{=} K_{n, 0}^{(5 / 2)}-\frac{2}{5 R} K_{n, 0}^{(3 / 2)}+\frac{2}{15 R^{2}} K_{n, 0}^{(1 / 2)} \text {. }
$$

Using this formula for $j=5$, we can demonstrate by analytic computations the validity of the following estimate for $j=5$ and $n=0,1,2,3$ :

$$
J_{\rho_{0}}\left[\tau, \breve{K}_{n, 0}^{\left(\alpha_{j}\right)}\right]= \begin{cases}A_{j}+\mathcal{O}\left(\frac{\rho^{2}}{R^{n+1}}\right) & n<\alpha_{j}-\alpha_{1} \\ A_{j}+\mathcal{O}\left(\rho^{2\left(\alpha_{1}-\alpha_{j}\right)+n+1}\left(\frac{\rho}{R}\right)^{n+1}\right) & n \geq \alpha_{j}-\alpha_{1}\end{cases}
$$

Formulas (76), (80), (82) show the same estimate for $j=3$ and $n=0,1,2$.

In the general case of resonance, for $j=2 \ell+1$, the modified QFM takes the form:

$$
\breve{K}_{n, 0}^{\left(\alpha_{j}\right)}=K_{n, 0}^{\left(\alpha_{j}\right)}+c_{1} K_{n, 0}^{\left(\alpha_{j}-1\right)}+\cdots+c_{\ell} K_{n, 0}^{\left(\alpha_{1}\right)} .
$$

4.2. Axisymmetric solution of a $3 \pi / 2$ V-notch with homogeneous Neumann BCs. We apply the QDFM for the extraction of the GEFIFs associated with a circular $3 \pi / 2$ V-Notch $\left(\omega=3 \pi / 2, \varphi_{1}=-\pi, \varphi_{2}=\pi / 2\right)$ and homogenous Neumann BCs. In this case the eigenvalues are $\alpha_{k}=0, \frac{2}{3}, \frac{4}{3}, \frac{6}{3}, \frac{8}{3} \cdots$, and the solution $\tau$ can be expressed as follows (see [12] for the expressions of $\phi_{\ell, k, i}$ ):

$$
\begin{aligned}
\tau & =A_{1} \rho^{2 / 3} \sum_{i=0}^{8}\left(\frac{\rho}{R}\right)^{i} \phi_{0,1, i}+A_{2} \rho^{4 / 3} \sum_{i=0}^{8}\left(\frac{\rho}{R}\right)^{i} \phi_{0,2, i}+A_{3} \rho^{2} \sum_{i=0}^{7}\left(\frac{\rho}{R}\right)^{i} \phi_{0,3, i} \\
& +A_{4} \rho^{8 / 3} \sum_{i=0}^{6}\left(\frac{\rho}{R}\right)^{i} \phi_{0,4, i}+\cdots+A_{14} \rho^{28 / 3} \phi_{0,14,0}+\mathcal{O}\left(\rho^{10}\right)
\end{aligned}
$$

The first terms $\phi_{0, j, 0}=\phi_{j}$ and $\psi_{0, j, 0}=\psi_{j}$ of the primal and dual singular functions are:

$$
\phi_{j}(\varphi)=\psi_{j}(\varphi)=\sin \frac{2 j \varphi}{3}-\frac{(-1)^{j}}{\sqrt{3}} \cos \frac{2 j \varphi}{3}, \quad j=1,2,4,5, \ldots
$$

Thus the quantity $D_{j}$ in (7) is equal to $\pi$. 
4.2.1. Extracting $A_{1}$. We first construct the QDF $K_{3,0}^{(2 / 3)} \stackrel{\text { def }}{=} K_{3,0}^{(2 / 3)}\left[B_{1}\right]$ with $B_{1}=\frac{3}{8 \pi^{2} R}$ according to (59):

$$
\begin{aligned}
K_{3,0}^{(2 / 3)} & =\frac{3}{8 \pi^{2} R} \rho^{-2 / 3}\left[\sin \frac{2 \varphi}{3}+\frac{1}{\sqrt{3}} \cos \frac{2 \varphi}{3}\right. \\
& +\left(\frac{5}{4 \sqrt{3}} \cos \frac{\varphi}{3}-\frac{5}{4} \sin \frac{\varphi}{3}-\frac{1}{4 \sqrt{3}} \cos \frac{5 \varphi}{3}-\frac{1}{4} \sin \frac{5 \varphi}{3}\right)\left(\frac{\rho}{R}\right) \\
+ & \left(-\frac{\sqrt{3}}{8} \cos \frac{2 \varphi}{3}-\frac{3}{8} \sin \frac{2 \varphi}{3}+\frac{\sqrt{3}}{32} \cos \frac{8 \varphi}{3}+\frac{3}{32} \sin \frac{8 \varphi}{3}\right)\left(\frac{\rho}{R}\right)^{2} \\
+ & \left(\frac{5 \sqrt{3}}{128} \cos \frac{\varphi}{3}-\frac{15}{128} \sin \frac{\varphi}{3}+\frac{7 \sqrt{3}}{128} \cos \frac{5 \varphi}{3}+\frac{21}{128} \sin \frac{5 \varphi}{3}\right. \\
& \left.\left.-\frac{5}{128 \sqrt{3}} \cos \frac{11 \varphi}{3}-\frac{5}{128} \sin \frac{11 \varphi}{3}+\frac{25}{128 \sqrt{3}} \cos \frac{7 \varphi}{3}-\frac{25}{128} \sin \frac{7 \varphi}{3}\right)\left(\frac{\rho}{R}\right)^{3}\right] .
\end{aligned}
$$

Of course that $K_{0,0}^{(2 / 3)}, K_{1,0}^{(2 / 3)}$ and $K_{2,0}^{(2 / 3)}$ are obtained if one neglects the corresponding dual shadows in (86).

We extract $A_{1}$ by the pseudo-surface independent integral (35) using $K_{0,0}^{(2 / 3)}, \ldots, K_{3,0}^{(2 / 3)}$. Combining (86) with the expansion (84), we check that in the V-notch case there is no improvement of the general convergence result (58). We obtain:

$$
J_{\rho_{0}}\left[\tau, K_{n, 0}^{(2 / 3)}\right]=A_{1}+\mathcal{O}\left(\frac{\rho_{0}}{R}\right)^{n+1}, \quad n=0,1,2,3
$$

the main contribution to the remainder being that of $A_{1}$.

4.2.2. Extracting $A_{2}$. For extraction of $A_{2}$ we construct $K_{3,0}^{(4 / 3)}$ as follows

$$
\begin{aligned}
K_{3,0}^{(4 / 3)} & =\frac{3}{16 \pi^{2} R} \rho^{-4 / 3}\left[\sin \frac{4 \varphi}{3}-\frac{1}{\sqrt{3}} \cos \frac{4 \varphi}{3}\right. \\
& +\left(\frac{1}{4 \sqrt{3}} \cos \frac{7 \varphi}{3}-\frac{1}{4} \sin \frac{7 \varphi}{3}-\frac{7}{4} \sin \frac{\varphi}{3}+\frac{7}{4 \sqrt{3}} \cos \frac{\varphi}{3}\right)\left(\frac{\rho}{R}\right) \\
& +\left(-\frac{\sqrt{3}}{4} \cos \frac{4 \varphi}{3}+\frac{3}{4} \sin \frac{4 \varphi}{3}-\frac{\sqrt{3}}{32} \cos \frac{10 \varphi}{3}+\frac{3}{32} \sin \frac{10 \varphi}{3}\right)\left(\frac{\rho}{R}\right)^{2} \\
& +\left(\frac{\sqrt{3}}{32} \cos \frac{\varphi}{3}-\frac{3}{32} \sin \frac{\varphi}{3}+\frac{17 \sqrt{3}}{128} \cos \frac{7 \varphi}{3}-\frac{51}{128} \sin \frac{7 \varphi}{3}+\right. \\
+ & \left.\left.\frac{5}{128 \sqrt{3}} \cos \frac{13 \varphi}{3}-\frac{5}{128} \sin \frac{13 \varphi}{3}+\frac{7}{16} \sin \frac{5 \varphi}{3}+\frac{7}{16 \sqrt{3}} \cos \frac{5 \varphi}{3}\right)\left(\frac{\rho}{R}\right)^{3}\right]
\end{aligned}
$$

The QDFs $K_{0,0}^{(4 / 3)}, K_{1,0}^{(4 / 3)}$ and $K_{2,0}^{(4 / 3)}$ are obtained if one neglects the corresponding dual shadows in (88).

We extract $A_{2}$ by the QDFM (35) using $K_{0,0}^{(4 / 3)}, \ldots, K_{3,0}^{(4 / 3)}$. According to (88) and (84) we find the same orders of convergence as in the general case (58):

$$
J_{\rho_{0}}\left[\tau, K_{0,0}^{(4 / 3)}\right]=A_{2}+\mathcal{O}\left[\rho_{0}^{-2 / 3}\left(\frac{\rho_{0}}{R}\right)^{n+1}\right] .
$$

Remark 12. We checked by several computations that for a circular $3 \pi / 2$ V-notch in an axisymmetric case, the GEFIFs $A_{j}$ may be extracted by the QDFM using $K_{n, 0}^{\left(\alpha_{j}\right)}$ according to the theoretical estimate (35). 
Remark 13. For extracting $A_{4}$ associated with $\alpha_{4}=8 / 3=\alpha_{1}+2$ the resonance situation occurs and the modified QDF must be used instead of the "regular" one. In this case of resonance we still obtain the theoretical estimate using the modified QDF for $n \geq \alpha_{j}-\alpha_{1}$ :

$$
J_{\rho_{0}}\left[\tau, \breve{K}_{n, 0}^{\left(\alpha_{j}\right)}\right]= \begin{cases}A_{j}+\mathcal{O}\left(\frac{\rho_{0}^{2}}{R^{n+1}}\right) & n<\alpha_{j}-\alpha_{1} \\ A_{j}+\mathcal{O}\left(\rho_{0}^{\alpha_{1}-\alpha_{j}}\left(\frac{\rho_{0}}{R}\right)^{n+1}\right) & n \geq \alpha_{j}-\alpha_{1}\end{cases}
$$

4.3. Extracting $A_{j}$ from $p$-FE solutions. Using the QDFM we extract $A_{j}$ from $p$-FE solutions because in general the exact solution $\tau$ is not known, but only its approximation $\tau_{\mathrm{FE}}$. A Visual Basic program was created for extracting the GEFIF's from the FE solution.

As an example, an axisymmetric FE model of a body with a circular singular edge is considered. Here the exact solution $\tau_{\mathrm{EX}}$ is approximated by the finite element solution $\tau_{\mathrm{FE}}$, and the integral $J_{\rho_{0}}\left[\tau_{\mathrm{FE}}, K_{n, 0}^{\left(\alpha_{j}\right)}\right]$ is computed numerically. $\tau_{\mathrm{FE}}$ is extracted on a torus surface which surrounds the circular singular edge (see Figure 3) and $K_{n, 0}^{\left(\alpha_{j}\right)}$ is computed analytically.

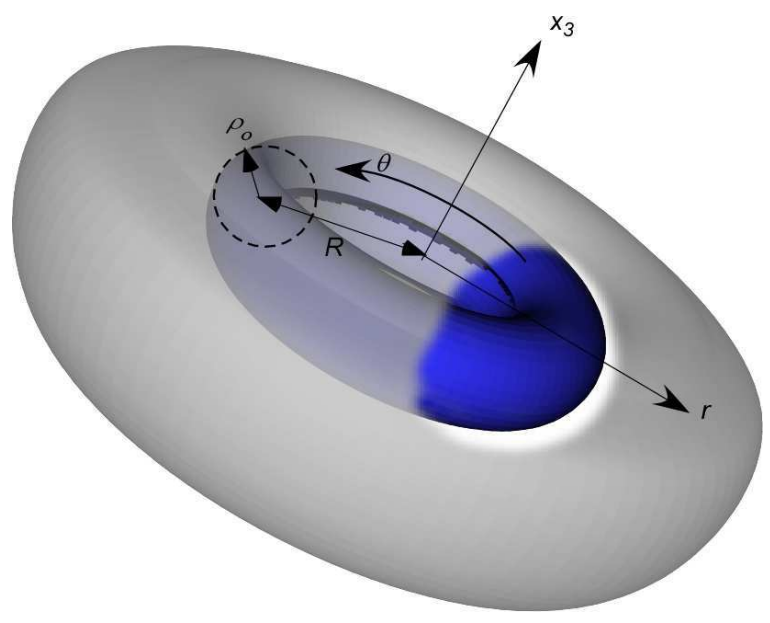

FIGURE 3. Domain with a circular singular edge and the integral surface.

Because the extracted values of $\tau_{\mathrm{FE}}$ are discrete, we compute the integral $J_{\rho_{0}}\left[\tau_{\mathrm{FE}}, K_{n, 0}^{\left(\alpha_{j}\right)}\right]$ by a Gauss quadrature:

$$
\begin{aligned}
& J_{\rho_{0}}\left[\tau_{\mathrm{FE}}, K_{n, 0}^{\left(\alpha_{j}\right)}\right]= \\
= & 2 \pi \int_{\varphi_{1}}^{\varphi_{2}}\left[K_{n, 0}^{\left(\alpha_{j}\right)}\left(\rho_{0}, \varphi\right) \times \partial_{\rho} \tau_{\mathrm{FE}}\left(\rho_{0}, \varphi\right)-\tau_{\mathrm{FE}}\left(\rho_{0}, \varphi\right) \times \partial_{\rho} K_{n, 0}^{\left(\alpha_{j}\right)}\left(\rho_{0}, \varphi\right)\right] \rho_{0} R\left(1+\frac{\rho_{0}}{R} \cos \varphi\right) d \varphi \\
= & 2 \pi \frac{\varphi_{2}-\varphi_{1}}{2} \int_{-1}^{1}\left[K_{n, 0}^{\left(\alpha_{j}\right)}\left(\rho_{0}, \varphi(\xi)\right) \times \partial_{\rho} \tau_{\mathrm{FE}}\left(\rho_{0}, \varphi(\xi)\right)-\tau_{\mathrm{FE}}\left(\rho_{0}, \varphi(\xi)\right) \times \partial_{\rho} K_{n, 0}^{\left(\alpha_{j}\right)}\left(\rho_{0}, \varphi(\xi)\right)\right] \\
& \times \rho_{0} R\left(1+\frac{\rho_{0}}{R} \cos (\varphi(\xi))\right) d \xi \\
= & \pi\left(\varphi_{2}-\varphi_{1}\right) \sum_{i=1}^{n_{\mathrm{GP}}} W_{i}\left[K_{n, 0}^{\left(\alpha_{j}\right)}\left(\rho_{0}, \varphi\left(\xi_{i}\right)\right) \times \partial_{\rho} \tau_{\mathrm{FE}}\left(\rho_{0}, \varphi\left(\xi_{i}\right)\right)-\tau_{\mathrm{FE}}\left(\rho_{0}, \varphi\left(\xi_{i}\right)\right) \times \partial_{\rho} K_{n, 0}^{\left(\alpha_{j}\right)}\left(\rho_{0}, \varphi\left(\xi_{i}\right)\right)\right] \\
& \times \rho_{0} R\left(1+\frac{\rho_{0}}{R} \cos \left(\varphi\left(\xi_{i}\right)\right)\right)
\end{aligned}
$$

with

$$
\varphi\left(\xi_{i}\right)=\frac{\varphi_{2}-\varphi_{1}}{2} \xi_{i}+\frac{\varphi_{2}+\varphi_{1}}{2},
$$


and $\xi_{i}$ and $W_{i}$ being abscissas and weights of the Gauss quadrature, and $n_{\mathrm{GP}}$ is the number of the Gauss points used for the numerical integration.

4.3.1. Sources of error in $A_{j}$ when extracted by $J_{\rho_{0}}\left[\tau_{\mathrm{FE}}, K_{n, 0}^{\left(\alpha_{j}\right)}\right]$. Extraction of $A_{j}$ by $J_{\rho_{0}}\left[\tau_{\mathrm{FE}}, K_{n, 0}^{\left(\alpha_{j}\right)}\right]$ involves three sources of errors:

(1) The error due to the truncation of $K_{n, 0}^{\left(\alpha_{j}\right)}$ :

Computing $A_{j}$ with $n$ being a finite number, a truncation error is introduced according to (58) and (60).

(2) The numerical error using $\tau_{\mathrm{FE}}$ instead of $\tau_{\mathrm{EX}}$ :

Using FE solutions for approximating $\tau_{\mathrm{EX}}$, a numerical error is included in our computations. We may estimate the numerical error by estimating the FE error. We expect that the error in the extracted GEFIFs is smaller than the error in the energy norm.

(3) The numerical integration error:

The values of $\tau_{\mathrm{FE}}$ are discrete therefore we use a Gauss quadrature for the evaluation of the integral (91). The Gaussian quadrature order $n_{\mathrm{GP}}$ determines the numerical integration error.

4.3.2. A specific example: a torus with a circular crack and axisymmetric Neumann BCs. The accuracy of the numerical procedure based on $p$-FE methods is firstly demonstrated on a simple problem of an inner cracked torus. Consider a torus with an axis (which is a circle) of a radius $R=1$, and a minor radius of $1 / 2$, i.e. the torus is defined by $\Omega=\{(\rho, \varphi, \theta) \mid 0<\rho<1 / 2,-\pi<\varphi<\pi, 0 \leq \theta<2 \pi\}$. The radial coordinate $r$ is bounded by $1 / 2=r_{1}<r<r_{2}<3 / 2$. A crack is inserted in the torus defined by $r<R=1, x_{3}=0$ (see Figure 4, Left). On the crack surfaces homogeneous Neumann boundary conditions are prescribed, whereas on

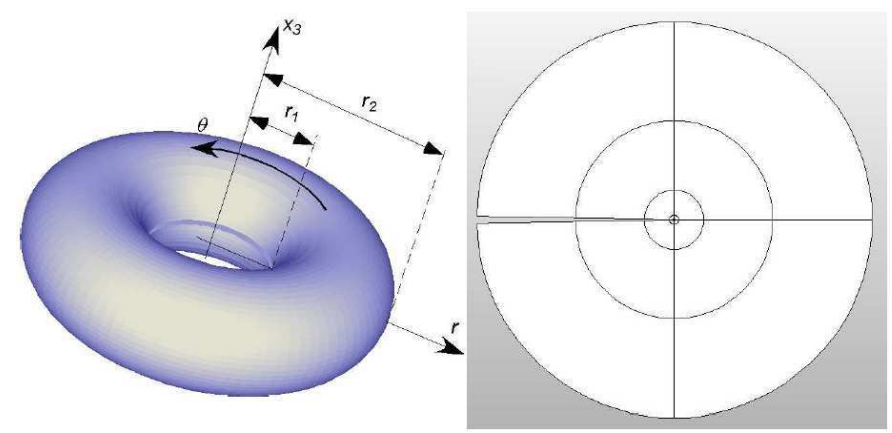

FIGURE 4. A torus with a circular crack and the axisymmetric finite element model.

the outer surface of the torus, $\rho=1 / 2,0 \leq \theta<2 \pi$ the trace of the exact solution (61) up to $\mathcal{O}\left(\rho^{10.5}\right)$ is prescribed as Dirichlet BCs, with $A_{0}=A_{2}=A_{4}=A_{6}=A_{7}=\cdots=0$, and $A_{1}=A_{3}=A_{5}=1$. Because an axisymmetric case is considered, we perform an axisymmetric FE analysis, using the mesh shown in Figure 4-Right. The analytic formula for the boundary conditions coincides with the exact solution up to an order of $(\rho / R)^{10.5}$ due to the truncation of series with respect to the index $i$.

The integral $J_{\rho_{0}}$ is computed using a quadrature of order $90\left(=n_{\mathrm{GP}}\right)$ and $\tau_{\mathrm{FE}}$ is extracted from a FE solution at $p=8$ having an error of $0.02 \%$ in energy norm.

We extract the first three EFIFs $A_{1}, A_{3}, A_{5}$ for different values of $\rho_{0}$ and consider an increasing number of dual shadow functions for the QDF $K_{n, 0}^{\left(\alpha_{j}\right)}$. For $A_{3}$ and $A_{5}$ the modified QDFs $\breve{K}_{n, 0}^{\left(\alpha_{j}\right)}$ are of course utilized.

The relative error as percentage of the "extracted $A_{j}^{\mathrm{FE}}$ " is defined as:

$$
e_{A_{j}} \%=100 \times\left|\frac{A_{j}^{\mathrm{FE}}-A_{j}^{\mathrm{Exact}}}{A_{j}^{\mathrm{Exact}}}\right|
$$


Figures 5-7 present the convergence of the extracted $A_{j}^{\mathrm{FE}}$. The convergence rates of the extracted $A_{j}^{\mathrm{FE}}$ match the convergence rates according to the estimates in (60).

As expected, the error in the extracted EFIFs is smaller than the errors in the FE solution. To quantify the

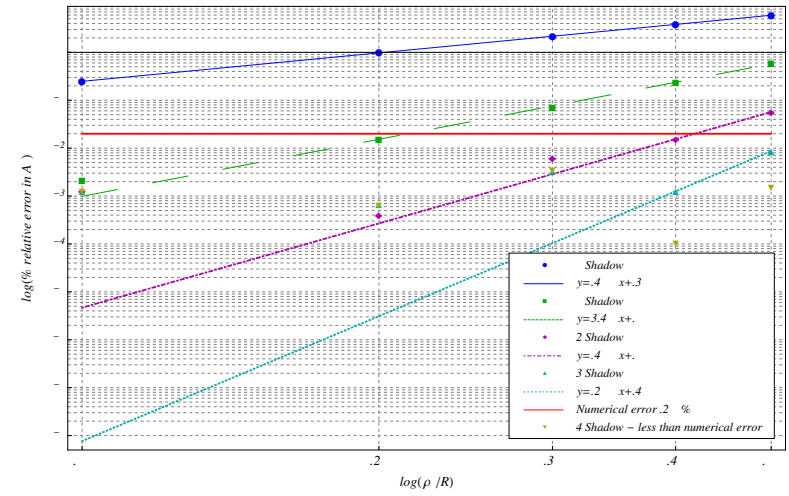

FiguRE 5. $e_{A_{1}} \%$ vs. $\log \left(\frac{\rho_{0}}{R}\right)$

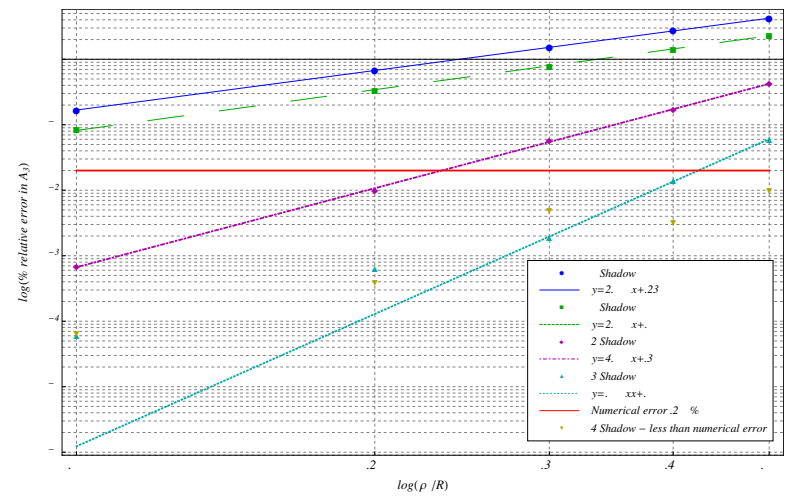

FIGURE 6. $e_{A_{3}} \%$ vs. $\log \left(\frac{\rho_{0}}{R}\right)$

integration error we monitor the relative error as percentage of $A_{1}, A_{3}, A_{5}$ extracted by $J_{\rho_{0}}\left[\tau_{\mathrm{FE}}, K_{2,0}^{\left(\alpha_{1}\right)}\right]$ and $J_{\rho_{0}}\left[\tau_{\mathrm{FE}}, \breve{K}_{2,0}^{\left(\alpha_{j}\right)}\right]$ when $j=3,5$ for different quadrature orders $n_{\mathrm{GP}}$. Table 2 presents a summary of these relative errors (percentage). One may observe that for $n_{\mathrm{GP}} \geq 32$ the error due to the Gauss quadrature is negligible.

4.3.3. A specific example: a penny shaped crack in a finite cylinder with axisymmetric Dirichlet BCs on the outer surface. Consider an axisymmetric FE model of a cylinder with a penny shaped crack. Homogeneous Neumann BCs are prescribed on the surface of the crack. On cylinder's outer surface Dirichlet BCs are prescribed, shown in Figure 8, given by

$$
\begin{aligned}
& \tau_{1}\left(r, x_{3}=1, \theta\right)=-155.4+3.055 r+181.8 r^{2}-34.82 r^{3}+6.43 r^{4}-1.27 r^{5}+0.22 r^{6} \\
& \tau_{2}\left(r, x_{3}=-1, \theta\right)=155.4-3.055 r-181.8 r^{2}+34.82 r^{3}-6.43 r^{4}+1.27 r^{5}-0.22 r^{6} \\
& \tau_{3}\left(r=2, x_{3}, \theta\right)=375.1 x_{3}
\end{aligned}
$$




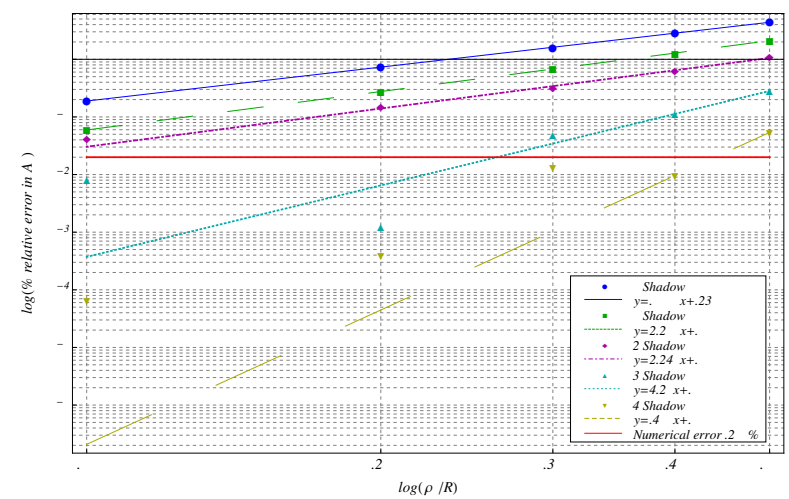

FIgURE 7. $e_{A_{5}} \%$ vs. $\log \left(\frac{\rho_{0}}{R}\right)$

TABLE 2. \% Relative error in $A_{j}$ for different quadrature orders for a circular crack with homogeneous Neumann BCs.

\begin{tabular}{|c||c|c|c|c|c|c|c|c|c|}
\hline \multicolumn{1}{|c||}{} & \multicolumn{3}{c|}{$\rho_{0} / R=1 / 2$} & \multicolumn{3}{c|}{$\rho_{0} / R=1 / 4$} & \multicolumn{3}{c|}{$\rho_{0} / R=1 / 10$} \\
\cline { 2 - 11 } & $n_{\mathrm{GP}}=10$ & $n_{\mathrm{GP}}=32$ & $n_{\mathrm{GP}}=90$ & $n_{\mathrm{GP}}=10$ & $n_{\mathrm{GP}}=32$ & $n_{\mathrm{GP}}=90$ & $n_{\mathrm{GP}}=10$ & $n_{\mathrm{GP}}=32$ & $n_{\mathrm{GP}}=90$ \\
\hline \hline$e_{A_{1}} \%$ & $4.52 \mathrm{E}+00$ & $9.24 \mathrm{E}-02$ & $5.68 \mathrm{E}-02$ & $1.84 \mathrm{E}-01$ & $1.77 \mathrm{E}-02$ & $2.78 \mathrm{E}-03$ & $1.99 \mathrm{E}-02$ & $2.67 \mathrm{E}-03$ & $1.24 \mathrm{E}-03$ \\
\hline$e_{A_{3}} \%$ & $1.30 \mathrm{E}+01$ & $4.73 \mathrm{E}-01$ & $4.28 \mathrm{E}-01$ & $1.74 \mathrm{E}+00$ & $5.89 \mathrm{E}-02$ & $2.38 \mathrm{E}-02$ & $2.80 \mathrm{E}-01$ & $1.38 \mathrm{E}-02$ & $6.94 \mathrm{E}-04$ \\
\hline$e_{A_{5}} \%$ & $4.38 \mathrm{E}+01$ & $1.15 \mathrm{E}+00$ & $1.10 \mathrm{E}+00$ & $1.88 \mathrm{E}+01$ & $1.60 \mathrm{E}-01$ & $2.34 \mathrm{E}-01$ & $8.57 \mathrm{E}+00$ & $7.89 \mathrm{E}-02$ & $4.20 \mathrm{E}-02$ \\
\hline
\end{tabular}

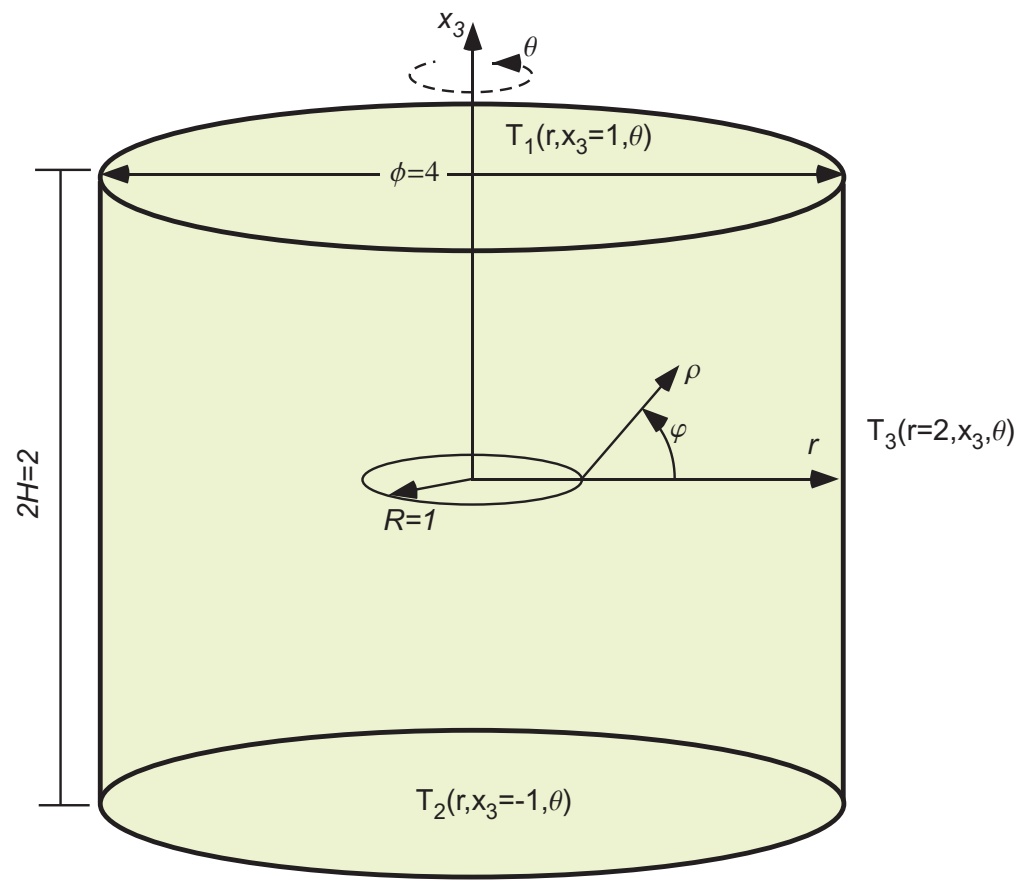

FIGURE 8. A cylinder with a penny-shaped crack and BCs. 
Because the domain and the boundary conditions (94)-(96) are $\theta$ independent, axisymmetric FE models were considered. To compute "benchmark values" of $A_{j}$ (because the exact values are unknown) we constructed a refined FE model as shown in Figure 9(a). These "benchmark $A_{j}$ " are extracted from the refined FE models by the pointwise contour integral method [1] implemented in StressCheck ${ }^{1}$ using 20 terms and a path radius of 0.01 . The error in the energy norm in the refined FE model is $0.05 \%$. In comparison, a coarse FE

(a)

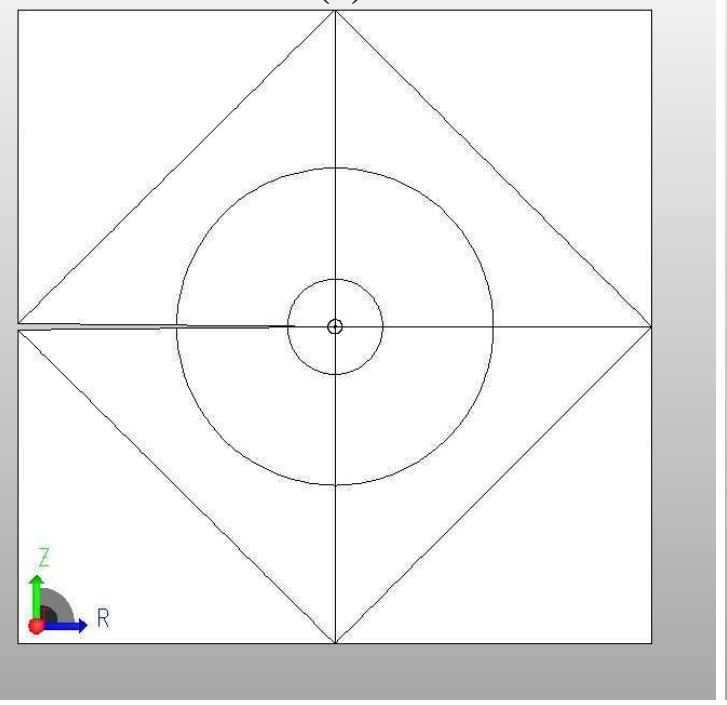

(b)

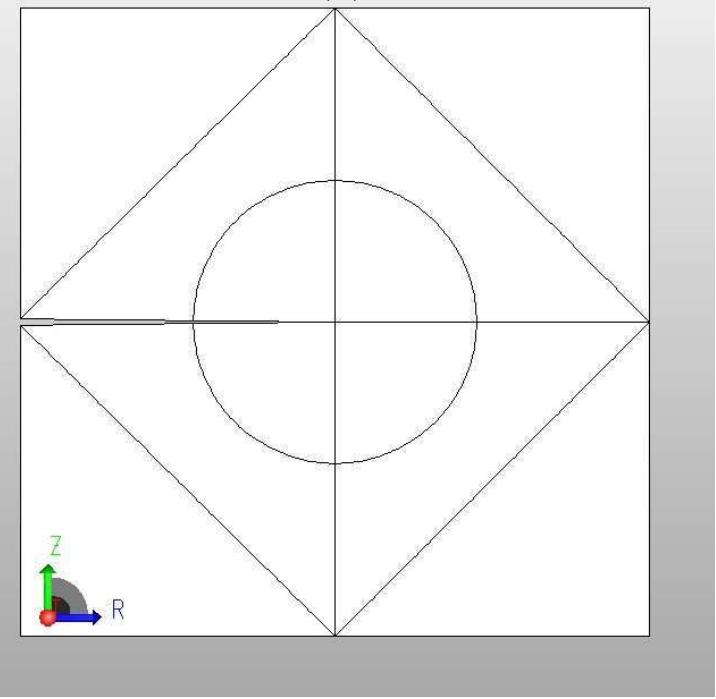

FIgURE 9. (a) A refined FE model, (b) A coarse FE model.

mesh shown in Figure 9(b) (the error in the energy norm is $0.35 \%$ ) was used for the extraction of the EFIFs by the QDFM. This is to demonstrate that no special refinements are required and a path away from the crack tip can be used for the QDFM. The benchmark values of the first three EFIFs extracted by the SC algorithm at $\rho_{0}=0.01$ are $A_{1}=30.15, A_{3}=116.41, A_{5}=91.02$.

The difference between the benchmark first three EFIFs and these computed by the QDFM at $\rho_{0}=1 / 2$ using 32 integration point is summarized in Table $3 . A_{j}$ for $j=3,5$ are extracted by $J_{\rho_{0}}\left[\tau, \breve{K}_{n, 0}^{\left(\alpha_{j}\right)}\right]$.

TABLE 3. Relative error (percentage) between $A_{j}$ extracted by SC using the refined mesh at $\rho_{0}=0.01$ and these extracted by the QDFM at $\rho_{0}=1 / 2$ using the coarse mesh, for different number of dual shadow functions.

\begin{tabular}{|l||c|c|c|c|c|}
\hline \multicolumn{1}{|c||}{} & \multicolumn{5}{c|}{ Number of dual shadow functions } \\
\cline { 2 - 6 } & $n=0$ & $n=1$ & $n=2$ & $n=3$ & $n=4$ \\
\hline \hline \% error in $A_{1}$ & 23.723 & 1.729 & 0.070 & 0.063 & 0.061 \\
\hline \% error in $A_{3}$ & 2.901 & 1.900 & 0.316 & 0.008 & 0.010 \\
\hline$\%$ error in $A_{5}$ & 1.122 & 1.452 & 0.964 & 0.178 & 0.055 \\
\hline
\end{tabular}

Remark 14. One may observe that extracting $A_{j}$ using the coarse FE mesh by $J_{\rho_{0}=1 / 2}\left[\tau_{\mathrm{FE}}, K_{4,0}^{\left(\alpha_{j}\right)}\right]$ results in very accurate values. The relative error in the extracted $A_{j} \mathrm{~s}$ is smaller than the FE relative error in energy norm.

\footnotetext{
${ }^{1}$ StressCheck is a trademark of ESRD, St. Louis, USA
} 


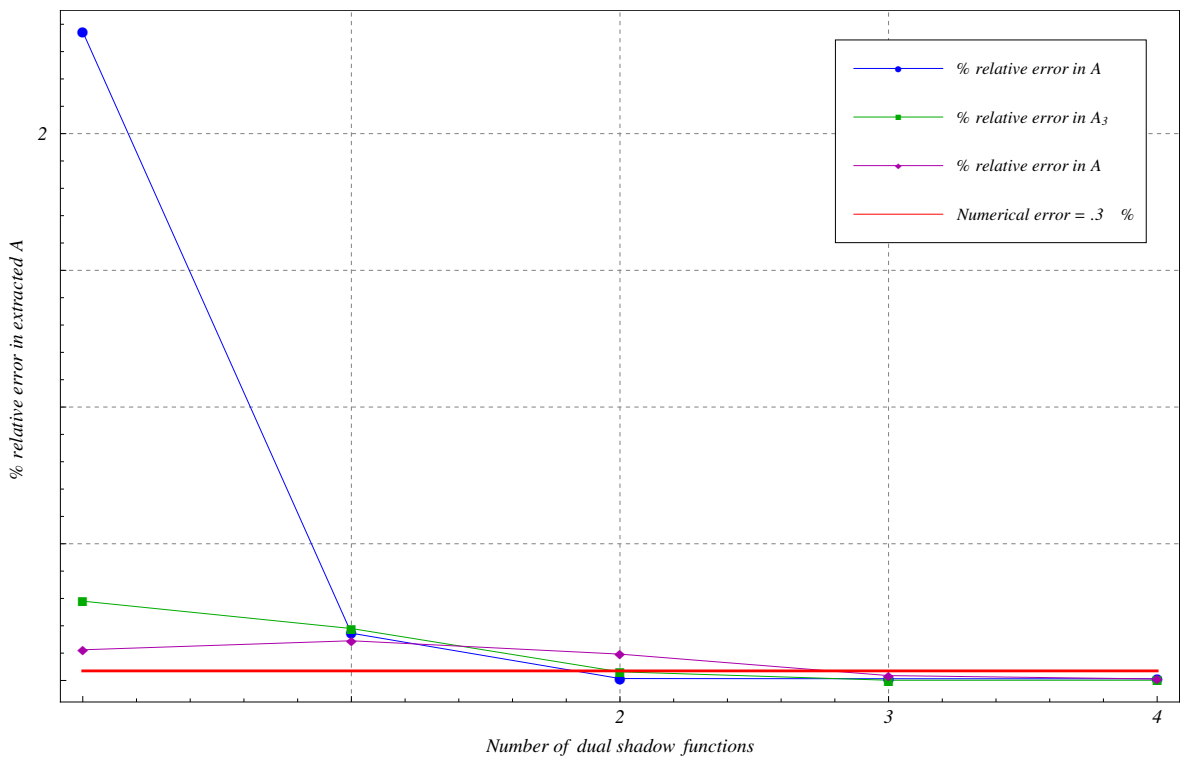

FIGURE 10. Relative error (percentage) between $A_{j}$ extracted by SC and by the QDFM versus the number of dual shadow functions in the QDF. 


\section{NONAXISYMMETRIC SOLUTIONS}

For nonaxisymmetric cases, the solution $\tau$ can be expressed as follows [15]:

$$
\tau(\rho, \varphi, \theta)=\sum_{k \geq 0} \sum_{\ell=0,2,4 \cdots} \partial_{\theta}^{\ell} A_{k}(\theta) \rho^{\alpha_{k}} \sum_{i \geq 0}\left(\frac{\rho}{R}\right)^{\ell+i} \phi_{\ell, k, i}(\varphi),
$$

and the QDFs will also depend on $\theta$ because of the functions $B_{j}(\theta)$ :

$$
K_{n, m}^{\left(\alpha_{j}\right)}(\rho, \varphi, \theta) \stackrel{\text { def }}{=} \sum_{h=0,2,4 \cdots}^{m} \partial_{\theta}^{h} B_{j}(\theta) \rho^{-\alpha_{j}} \sum_{f=0}^{n}\left(\frac{\rho}{R}\right)^{h+f} \psi_{h, j, f}(\varphi) .
$$

The explicit ODEs for the determination of the dual eigenfunctions and dual shadow functions, $\psi_{h, j, f}(\varphi)$, are obtained from (22):

For $h=0: \quad$ Equations (53)-(55) for the axisymmetric case hold.

For $h=2,4,6 \cdots, \quad f \geq 0$ :

$$
\begin{aligned}
\left(-\alpha_{j}+\right. & f+h)^{2} \psi_{h, j, f}+\psi_{h, j, f}^{\prime \prime}= \\
& -\left(h+f-\alpha_{j}-1\right)\left[2\left(h+f-\alpha_{j}\right)-1\right] \cos \varphi \psi_{h, j,(f-1)}+\sin \varphi \psi_{h, j,(f-1)}^{\prime} \\
& -2 \cos \varphi \psi_{h, f,(f-1)}^{\prime \prime}-\left(h-\alpha_{j}+f-2\right)\left(h-\alpha_{j}+f-1\right) \cos ^{2} \varphi \psi_{h, j,(f-2)} \\
& +\cos \varphi \sin \varphi \psi_{h, j,(f-2)}^{\prime}-\cos ^{2} \varphi \psi_{h, j,(f-2)}^{\prime \prime}-\psi_{(h-2), j, f}
\end{aligned}
$$

with homogeneous BCs,

$$
\begin{array}{rll}
\psi_{h, j, f}(\varphi) & =0, \text { on } \varphi=\varphi_{1}, \varphi_{2}, & \text { Homogeneous Drichlet BCs, } \\
\partial_{\varphi} \psi_{h, j, f}(\varphi) & =0, \text { on } \varphi=\varphi_{1}, \varphi_{2}, \quad \text { Homogeneous Neumann BCs. }
\end{array}
$$

For a circular closed edge $(\theta \in[0,2 \pi])$, by elliptic regularity along the edge So they can be expanded as a convergent Fourier series:

$$
A_{k}(\theta)=a_{k_{0}}+\sum_{p=1}^{\infty} a_{k_{2 p-1}} \cos (p \theta)+\sum_{p=1}^{\infty} a_{k_{2 p}} \sin (p \theta) .
$$

In this case the QDFM amounts to extract a finite number of the coefficients $a_{k_{q}}$ with $q=0, \ldots, Q$ and to approximate $A_{k}$ by its truncated Fourier series

$$
A_{k}^{[Q]}(\theta) \stackrel{\text { def }}{=} a_{k_{0}}+\sum_{1 \leq 2 p-1 \leq Q} a_{k_{2 p-1}} \cos (p \theta)+\sum_{2 \leq 2 p \leq Q} a_{k_{2 p}} \sin (p \theta) .
$$

5.1. $B_{j}(\theta)$ for a nonaxisymmetric case. For extracting the coefficients $a_{j_{q}}$, we choose $B_{j_{q}}(\theta)$ orthogonal to all functions in (103) except the one that multiplies $a_{j_{q}}$, i.e., $B_{j_{q}}(\theta)$ is chosen as

$$
B_{j_{q}}(\theta)=b_{j_{q}} \cos (q \theta) \text { for even } q \text { or } B_{j_{q}}(\theta)=b_{j_{q}} \sin (q \theta) \text { for odd } q .
$$

Substituting in (35) one obtains:

$$
\begin{aligned}
J_{\rho_{0}}\left[\tau, K_{n, m}^{\left(\alpha_{j}\right)}\left[B_{j_{q}}\right]\right] & =\left(\int_{0}^{2 \pi} A_{j} B_{j_{q}} d \theta\right)\left(2 \alpha_{j} R \int_{\varphi_{1}}^{\varphi_{2}} \phi_{j}^{2} d \varphi\right)+\mathcal{O}\left(\rho^{\alpha_{1}-\alpha_{j}+1+\min \{n, m\}}\right) \\
& =a_{j_{q}}+\mathcal{O}\left(\rho^{\alpha_{1}-\alpha_{j}+1+\min \{n, m\}}\right)
\end{aligned}
$$

where for a circular closed edge of radius $R, b_{j_{q}}$ is given by:

$$
\begin{array}{rlrl}
b_{j_{0}} & =\frac{1}{4 \alpha_{j} \pi^{2} R}, & & q=0 \\
b_{j_{q}}=\frac{1}{2 \alpha_{j} \pi^{2} R}, & & q \neq 0
\end{array}
$$


5.2. A circular crack with homogeneous Neumann BCs. Following section 4.1, we represent $\tau$ up to a power of $\rho^{21 / 2}$ (the explicit expressions for $\phi_{\ell, k, i}$ are provided in [12]):

$$
\begin{aligned}
\tau & =A_{1}(\theta) \rho^{1 / 2} \sum_{i=0}^{10}\left(\frac{\rho}{R}\right)^{i} \phi_{0,1, i}+\partial_{\theta}^{2} A_{1}(\theta) \rho^{1 / 2}\left(\frac{\rho}{R}\right)^{2} \sum_{i=0}^{8}\left(\frac{\rho}{R}\right)^{i} \phi_{2,1, i} \\
& +\partial_{\theta}^{4} A_{1}(\theta) \rho^{1 / 2}\left(\frac{\rho}{R}\right)^{4} \sum_{i=0}^{6}\left(\frac{\rho}{R}\right)^{i} \phi_{4,1, i}+\cdots+\partial_{\theta}^{10} A_{1}(\theta) \rho^{1 / 2}\left(\frac{\rho}{R}\right)^{10} \phi_{10,1,0} \\
& +A_{2}(\theta) \rho \sum_{i=0}^{10}\left(\frac{\rho}{R}\right)^{i} \phi_{0,2, i}+\partial_{\theta}^{2} A_{2}(\theta) \rho\left(\frac{\rho}{R}\right)^{2} \sum_{i=0}^{8}\left(\frac{\rho}{R}\right)^{i} \phi_{2,2, i} \\
& +\partial_{\theta}^{4} A_{2}(\theta) \rho\left(\frac{\rho}{R}\right)^{4} \sum_{i=0}^{6}\left(\frac{\rho}{R}\right)^{i} \phi_{4,2, i}+\cdots+\partial_{\theta}^{10} A_{2}(\theta) \rho\left(\frac{\rho}{R}\right)^{10} \phi_{10,2,0} \\
& +\cdots+A_{21} \rho^{21 / 2} \phi_{0,21,0}
\end{aligned}
$$

To extract any of the coefficients $a_{1_{q}}$ of the first GSIF $A_{1}(\theta)$, a special quasi-dual function $K_{n, m}^{(1 / 2)}\left[B_{1_{q}}\right]$ needs to be employed. For $n=1$ and $m=2$ we have calculated

$$
\begin{aligned}
K_{1,2}^{(1 / 2)}\left[B_{1_{q}}\right]= & B_{1_{q}}(\theta) \rho^{-1 / 2}\left[\sin \frac{\varphi}{2}-\frac{1}{4} \sin \frac{3 \varphi}{2}\left(\frac{\rho}{R}\right)\right] \\
& +\partial_{\theta}^{2} B_{1_{q}}(\theta) \rho^{-1 / 2}\left[-\frac{1}{2} \sin \frac{\varphi}{2}\left(\frac{\rho}{R}\right)^{2}+\left(-\frac{1}{4} \sin \frac{\varphi}{2}+\frac{3}{8} \sin \frac{3 \varphi}{2}\right)\left(\frac{\rho}{R}\right)^{3}\right]
\end{aligned}
$$

For example for $B_{1_{0}}(\theta)=b_{1_{0}}$, the QDF in (109) is:

$$
K_{1,2}^{(1 / 2)}\left[B_{1_{0}}\right]=b_{1_{0}}(\theta) \rho^{-1 / 2}\left[\sin \frac{\varphi}{2}-\frac{1}{4} \sin \frac{3 \varphi}{2}\left(\frac{\rho}{R}\right)\right]
$$

which coincides with $K_{1,2}^{(1 / 2)}$ given by (65).

For $B_{1_{1}}(\theta)=b_{1_{1}} \cos (\theta)$ the QDF in (109) is:

$$
\begin{aligned}
K_{1,2}^{(1 / 2)}\left[B_{1_{1}}\right]= & b_{1_{1}} \cos (\theta) \rho^{-1 / 2}\left[\sin \frac{\varphi}{2}-\frac{1}{4} \sin \frac{3 \varphi}{2}\left(\frac{\rho}{R}\right)\right] \\
& -b_{1_{1}} \cos (\theta) \rho^{-1 / 2}\left[-\frac{1}{2} \sin \frac{\varphi}{2}\left(\frac{\rho}{R}\right)^{2}+\left(-\frac{1}{4} \sin \frac{\varphi}{2}+\frac{3}{8} \sin \frac{3 \varphi}{2}\right)\left(\frac{\rho}{R}\right)^{3}\right]
\end{aligned}
$$

and so on for $q=2, \cdots$ (in the sequel, for our FE computations, we go up to $q=8$, approximating nine coefficients). 
Substituting (108) and (109) in (35) we calculate:

$$
\begin{aligned}
J_{\rho_{0}}[\tau \quad & \left., K_{1,2}^{(1 / 2)}\left[B_{1_{q}}\right]\right]= \\
& \int_{0}^{2 \pi} \pi R \times\left\{\left[\left(1-\frac{3 \rho_{0}^{5}}{1024 R^{5}}+\cdots\right) A_{1}(\theta)+\left(\frac{3 \rho_{0}^{6}}{1120 R^{5}}+\cdots\right) A_{3}(\theta)\right.\right. \\
& +\left(-\frac{3 \rho_{0}^{4}}{32 R^{2}}+\cdots\right) A_{5}(\theta)+\cdots+\left(-\frac{\rho_{0}^{2}}{2 R^{2}}-\frac{\rho_{0}^{3}}{4 R^{3}}-\frac{5 \rho_{0}^{4}}{24 R^{4}}+\cdots\right) A_{1}^{\prime \prime}(\theta) \\
& \left.+\left(\frac{\rho_{0}^{4}}{4 R^{3}}+\frac{3 \rho_{0}^{5}}{32 R^{4}}+\cdots\right) A_{3}^{\prime \prime}(\theta)+\left(-\frac{47 \rho_{0}^{6}}{448 R^{4}}+\cdots\right) A_{5}^{\prime \prime}(\theta)+\cdots\right] \times B_{1_{q}}(\theta) \\
& +\left[\left(\frac{\rho_{0}^{2}}{2 R^{2}}+\frac{\rho_{0}^{3}}{4 R^{3}}-\frac{29 \rho_{0}^{4}}{48 R^{4}}+\cdots\right) A_{1}(\theta)+\left(-\frac{\rho_{0}^{4}}{4 R^{3}}+\frac{5 \rho_{0}^{5}}{32 R^{4}}+\cdots\right) A_{3}(\theta)\right. \\
& +\left(-\frac{7 \rho_{0}^{6}}{64 R^{4}}+\cdots\right) A_{5}(\theta)+\cdots+\left(\frac{\rho_{0}^{4}}{12 R^{4}}+\cdots\right) A_{1}^{\prime \prime}(\theta)+\left(-\frac{\rho_{0}^{6}}{10 R^{5}}+\cdots\right) A_{3}^{\prime \prime}(\theta) \\
& \left.\left.+\left(\frac{57 \rho_{0}^{8}}{896 R^{6}}+\cdots\right) A_{5}^{\prime \prime}(\theta)+\cdots\right] \times B_{1_{q}^{\prime \prime}}^{\prime \prime}(\theta)\right\} d \theta .
\end{aligned}
$$

The coefficient $a_{1_{0}}$, for example, is obtained by using $B_{1_{0}}(\theta)=b_{1_{0}}=\frac{1}{2 \pi^{2} R}$ in (111),

$$
J_{\rho_{0}}\left[\tau, K_{1,2}^{(1 / 2)}\left[B_{1_{0}}\right]\right]=a_{1_{0}}+\mathcal{O}\left(\rho_{0}^{2}\left(\frac{\rho_{0}}{R}\right)^{2}\right)
$$

which is coherent with (67) in the axisymmetric case.

For $a_{1_{1}}$ we need $B_{1_{1}}(\theta)=b_{1_{1}} \cos (\theta)=\frac{1}{\pi^{2} R} \cos (\theta)$, so (111) becomes,

$$
J_{\rho_{0}}\left[\tau, K_{1,2}^{(1 / 2)}\left[B_{1_{1}}\right]\right]=a_{1_{1}}+\mathcal{O}\left(\rho_{0}^{2}\left(\frac{\rho_{0}}{R}\right)^{2}\right)
$$

Similarly, we extract $a_{1_{q}}$ by computing $J_{\rho_{0}}\left[\tau, K_{n, m}^{\left(\alpha_{j}\right)}\left[B_{1_{q}}\right]\right]$ for different $n$ and $m$ with different $B_{1_{q}}(\theta)$ and summarize the remainder of $J_{\rho_{0}}\left[\tau, K_{n, m}^{\left(\alpha_{1}\right)}\left[B_{1_{q}}\right]\right]$ in Appendix B in Tables 13-15.

Based on the results in Tables 13-15 and similar calculations we conclude that:

$$
J_{\rho_{0}}\left[\tau, K_{n, m}^{\left(\alpha_{j}\right)}\left[B_{j_{q}}\right]\right]=a_{j_{q}}+\mathcal{O}\left\{\rho_{0}^{2\left(\alpha_{1}-\alpha_{j}\right)+n+1}\left(\frac{\rho_{0}}{R}\right)^{n+1}+\rho_{0}^{\alpha_{1}-\alpha_{j}}\left(\frac{\rho_{0}}{R}\right)^{m+2}\right\} .
$$

Observing that the second term in the right hand side is larger compared to the first term, then for the optimal remainder, $m=n-1$, and (114) gives back the estimate in (35).

In the case of resonance, a modified QDF should be used, and instead of (114) one has:

$$
J_{\rho_{0}}\left[\tau, \breve{K}_{n, m}^{\left(\alpha_{j}\right)}\left[B_{j_{q}}\right]\right]= \begin{cases}a_{j_{q}}+\mathcal{O}\left[\left(\frac{\rho_{0}^{2}}{R^{n+1}}\right)+\rho_{0}^{\alpha_{1}-\alpha_{j}}\left(\frac{\rho_{0}}{R}\right)^{m+2}\right], & n<\alpha_{j}-\alpha_{1} \\ a_{j_{q}}+\mathcal{O}\left[\left(\rho_{0}^{2\left(\alpha_{1}-\alpha_{j}\right)+n+1}\left(\frac{\rho_{0}}{R}\right)^{n+1}\right)+\rho_{0}^{\alpha_{1}-\alpha_{j}}\left(\frac{\rho_{0}}{R}\right)^{m+2}\right], & n \geq \alpha_{j}-\alpha_{1}\end{cases}
$$


5.3. A $3 \pi / 2$ V-notch with homogeneous Neumann BCs. For a V-Notch $\varphi \in[-\pi, \pi / 2]$ with a general BCs, the solution $\tau$ can be presented as (see [12] for the explicit functions $\phi_{i, j, k}$ ):

$$
\begin{aligned}
\tau & =A_{1}(\theta) \rho^{2 / 3} \sum_{i=0}^{10}\left(\frac{\rho}{R}\right)^{i} \phi_{0,1, i}+\partial_{\theta}^{2} A_{1}(\theta) \rho^{2 / 3} \sum_{i=0}^{8}\left(\frac{\rho}{R}\right)^{2+i} \phi_{2,1, i}+\cdots+\partial_{\theta}^{10} A_{1}(\theta) \rho^{2 / 3}\left(\frac{\rho}{R}\right)^{10} \phi_{10,1,0} \\
& +A_{2}(\theta) \rho^{4 / 3} \sum_{i=0}^{10}\left(\frac{\rho}{R}\right)^{i} \phi_{0,2, i}+\partial_{\theta}^{2} A_{2}(\theta) \rho^{4 / 3} \sum_{i=0}^{8}\left(\frac{\rho}{R}\right)^{2+i} \phi_{2,2, i}+\cdots+\partial_{\theta}^{10} A_{2}(\theta) \rho^{4 / 3}\left(\frac{\rho}{R}\right)^{10} \phi_{10,2,0} \\
& +A_{3}(\theta) \rho^{2} \sum_{i=0}^{9}\left(\frac{\rho}{R}\right)^{i} \phi_{0,3, i}+\partial_{\theta}^{2} A_{3}(\theta) \rho^{2} \sum_{i=0}^{7}\left(\frac{\rho}{R}\right)^{2+i} \phi_{2,3, i}+\cdots+\partial_{\theta}^{8} A_{3}(\theta) \rho^{2} \sum_{i=0}^{1}\left(\frac{\rho}{R}\right)^{8+i} \phi_{8,3, i} \\
& +A_{4}(\theta) \rho^{8 / 3} \sum_{i=0}^{8}\left(\frac{\rho}{R}\right)^{i} \phi_{0,4, i}+\partial_{\theta}^{2} A_{4}(\theta) \rho^{8 / 3} \sum_{i=0}^{6}\left(\frac{\rho}{R}\right)^{2+i} \phi_{2,4, i}+\cdots+\partial_{\theta}^{8} A_{4}(\theta) \rho^{8 / 3} \sum_{i=0}^{1}\left(\frac{\rho}{R}\right)^{8+i} \phi_{10,4, i}
\end{aligned}
$$

The quasi-dual function $K_{0,2}^{(2 / 3)}$, for $n=0$ and $m=2$, for example is,

$$
\begin{aligned}
K_{0,2}^{(2 / 3)}= & B_{1_{q}}(\theta) \rho^{-2 / 3}\left(\sin \frac{2 \varphi}{3}+\frac{1}{\sqrt{3}} \cos \frac{2 \varphi}{3}\right) \\
& +\partial_{\theta}^{2} B_{1_{q}}(\theta) \rho^{-2 / 3}\left(\frac{\rho}{R}\right)^{2}\left(-\frac{1}{4} \sqrt{3} \cos \frac{2 \varphi}{3}-\frac{3}{4} \sin \frac{2 \varphi}{3}\right)
\end{aligned}
$$

Following the same procedure as in subsection 5.2, for extracting $a_{1_{1}}$ for example, $B_{1_{1}}(\theta)$ is chosen to be

$$
B_{1_{1}}(\theta)=\frac{6}{8 \pi^{2} R} \cos \theta
$$

and (116), (117) and (118) are used

$$
\begin{aligned}
J_{\rho_{0}\left[\tau, K_{0,2}^{(2 / 3)}\left[B_{1_{1}}\right]\right]=} & \left(1+\frac{10 \rho_{0}}{21 \pi R}+\cdots\right) a_{1_{1}}+\left(\frac{2 \rho_{0}^{5 / 3}}{3 \pi R}+\cdots\right) a_{2_{1}} \\
& +\left(\frac{15 \sqrt{3} \rho_{0}^{7 / 3}}{77 \pi R}+\cdots\right) a_{3_{1}}+\left(\frac{10 \rho_{0}^{3}}{117 \pi R}+\cdots\right) a_{4_{1}} \\
= & a_{1_{1}}+\mathcal{O}\left(\frac{\rho_{0}}{R}\right)
\end{aligned}
$$

Likewise, we compute $J_{\rho_{0}}\left[\tau, K_{n, m}^{(2 / 3)}\left[B_{1_{q}}\right]\right]$ for different $n$ and $m$ using (116) and (117) for different $B_{1_{q}}(\theta)$. The remainder is presented in Tables 4-6.

TABLE 4. The remainder of $J_{\rho_{0}}\left[\tau, K_{n, m}^{(2 / 3)}\left[B_{1_{0}}\right]\right]$

\begin{tabular}{cccc}
\hline \multicolumn{4}{c}{$B_{1_{0}}(\theta)=b_{1_{0}}=\frac{3}{8 \pi^{2} R}$} \\
\hline$m=0$ & $m=2$ & $m=4$ \\
\hline$n=0$ & $a_{1_{0}} \mathcal{O}\left(\frac{\rho_{0}}{R}\right)$ & $a_{1_{0}} \mathcal{O}\left(\frac{\rho_{0}}{R}\right)$ & $a_{1_{0}} \mathcal{O}\left(\frac{\rho_{0}}{R}\right)$ \\
$n=1$ & $a_{1_{0}} \mathcal{O}\left(\frac{\rho_{0}}{R}\right)^{2}$ & $a_{1_{0}} \mathcal{O}\left(\frac{\rho_{0}}{R}\right)^{2}$ & $a_{1_{0}} \mathcal{O}\left(\frac{\rho_{0}}{R}\right)^{2}$ \\
$n=2$ & $a_{1_{0}} \mathcal{O}\left(\frac{\rho_{0}}{R}\right)^{3}$ & $a_{1_{0}} \mathcal{O}\left(\frac{\rho_{0}}{R}\right)^{3}$ & $a_{1_{0}} \mathcal{O}\left(\frac{\rho_{0}}{R}\right)^{3}$ \\
$n=3$ & $a_{1_{0}} \mathcal{O}\left(\frac{\rho_{0}}{R}\right)^{4}$ & $a_{1_{0}} \mathcal{O}\left(\frac{\rho_{0}}{R}\right)^{4}$ & $a_{1_{0}} \mathcal{O}\left(\frac{\rho_{0}}{R}\right)^{4}$ \\
$n=4$ & $a_{1_{0}} \mathcal{O}\left(\frac{\rho_{0}}{R}\right)^{5}$ & $a_{1_{0}} \mathcal{O}\left(\frac{\rho_{0}}{R}\right)^{5}$ & $a_{1_{0}} \mathcal{O}\left(\frac{\rho_{0}}{R}\right)^{5}$ \\
\hline
\end{tabular}


TABLE 5. The remainder of $J_{\rho_{0}}\left[\tau, K_{n, m}^{(2 / 3)}\left[B_{1_{1}}\right]\right]$

\begin{tabular}{cccc}
\hline \multicolumn{4}{c}{$B_{1_{1}}(\theta)=b_{1_{1}}=\frac{6}{8 \pi^{2} R} \cos \theta$} \\
\hline$m=0$ & $m=2$ & $m=4$ \\
\hline$n=0$ & $a_{1_{0}} \mathcal{O}\left(\frac{\rho_{0}}{R}\right)$ & $a_{1_{0}} \mathcal{O}\left(\frac{\rho_{0}}{R}\right)$ & $a_{1_{0}} \mathcal{O}\left(\frac{\rho_{0}}{R}\right)$ \\
$n=1$ & $a_{1_{0}} \mathcal{O}\left(\frac{\rho_{0}}{R}\right)^{2}$ & $a_{1_{0}} \mathcal{O}\left(\frac{\rho_{0}}{R}\right)^{2}$ & $a_{1-0} \mathcal{O}\left(\frac{\rho_{0}}{R}\right)^{2}$ \\
$n=2$ & $a_{1_{0}} \mathcal{O}\left(\frac{\rho_{0}}{R}\right)^{2}$ & $a_{1_{0}} \mathcal{O}\left(\frac{\rho_{0}}{R}\right)^{3}$ & $a_{1_{0}} \mathcal{O}\left(\frac{\rho_{0}}{R}\right)^{3}$ \\
$n=3$ & $a_{1_{0}} \mathcal{O}\left(\frac{\rho_{0}}{R}\right)^{2}$ & $a_{1_{0}} \mathcal{O}\left(\frac{\rho_{0}}{R}\right)^{4}$ & $a_{1_{0}} \mathcal{O}\left(\frac{\rho_{0}}{R}\right)^{4}$ \\
$n=4$ & $a_{1_{0}} \mathcal{O}\left(\frac{\rho_{0}}{R}\right)^{2}$ & $a_{1_{0}} \mathcal{O}\left(\frac{\rho_{0}}{R}\right)^{4}$ & $a_{1_{0}} \mathcal{O}\left(\frac{\rho_{0}}{R}\right)^{5}$ \\
\hline
\end{tabular}

TABLE 6. The remainder of $J_{\rho_{0}}\left[\tau, K_{n, m}^{(2 / 3)}\left[B_{1_{3}}\right]\right]$

\begin{tabular}{cccc}
\hline \multicolumn{4}{c}{$B_{1_{3}}(\theta)=b_{1_{3}}=\frac{6}{8 \pi^{2} R} \cos 2 \theta$} \\
\hline$m=0$ & $m=2$ & $m=4$ \\
\hline$n=0$ & $a_{1_{0}} \mathcal{O}\left(\frac{\rho_{0}}{R}\right)$ & $a_{1_{0}} \mathcal{O}\left(\frac{\rho_{0}}{R}\right)$ & $a_{1_{0}} \mathcal{O}\left(\frac{\rho_{0}}{R}\right)$ \\
$n=1$ & $a_{1_{0}} \mathcal{O}\left(\frac{\rho_{0}}{R}\right)^{2}$ & $a_{1_{0}} \mathcal{O}\left(\frac{\rho_{0}}{R}\right)^{2}$ & $a_{1_{0}} \mathcal{O}\left(\frac{\rho_{0}}{R}\right)^{2}$ \\
$n=2$ & $a_{1_{0}} \mathcal{O}\left(\frac{\rho_{0}}{R}\right)^{2}$ & $a_{1_{0}} \mathcal{O}\left(\frac{\rho_{0}}{R}\right)^{3}$ & $a_{1_{0}} \mathcal{O}\left(\frac{\rho_{0}}{R}\right)^{3}$ \\
$n=3$ & $a_{1_{0}} \mathcal{O}\left(\frac{\rho_{0}}{R}\right)^{2}$ & $a_{1_{0}} \mathcal{O}\left(\frac{\rho_{0}}{R}\right)^{4}$ & $a_{1_{0}} \mathcal{O}\left(\frac{\rho_{0}}{R}\right)^{4}$ \\
$n=4$ & $a_{1_{0}} \mathcal{O}\left(\frac{\rho_{0}}{R}\right)^{2}$ & $a_{1_{0}} \mathcal{O}\left(\frac{\rho_{0}}{R}\right)^{4}$ & $a_{1_{0}} \mathcal{O}\left(\frac{\rho_{0}}{R}\right)^{5}$ \\
\hline
\end{tabular}

The results in Tables 4-6 follow the estimate in (35):

$$
J_{\rho_{0}}\left[\tau, K_{n, m}^{\left(\alpha_{j}\right)}\left[B_{j_{q}}\right]\right]=a_{j_{q}}+\mathcal{O}\left\{\rho_{0}^{\alpha_{1}-\alpha_{j}}\left[\left(\frac{\rho_{0}}{R}\right)^{n+1}+\left(\frac{\rho_{0}}{R}\right)^{m+2}\right]\right\}
$$

5.4. Extracting $a_{j_{q}}(\theta)$ from $p$-FE nonaxisymmetric solutions. The use of the QDFM in conjunction with $p$-FE nonaxisymmetric models for extracting $A_{j}(\theta)$ is examined. Consider a 3-D FE model of a body with a circular singular edge (for example: a penny shaped crack). We compute $J_{\rho_{0}}\left[\tau_{\mathrm{FE}}, K_{n, m}^{\left(\alpha_{j}\right)}\left[B_{j_{q}}\right]\right]$ where $\tau_{\mathrm{FE}}$ is extracted from the FE solution over a torus surface which surrounds the circular singular edge. $K_{n, m}^{\left(\alpha_{j}\right)}$ is computed analytically. Because the values of $\tau_{\mathrm{FE}}$ are discrete we compute the integral $J_{\rho_{0}}\left[\tau_{\mathrm{FE}}, K_{n, m}^{\left(\alpha_{j}\right)}\left[B_{j_{q}}\right]\right]$ by a Gauss quadrature.

$$
\begin{aligned}
J_{\rho_{0}}\left[\tau_{\mathrm{FE}}, K_{n, m}^{(\alpha, j)}\left[B_{j_{q}}\right]\right]= & \int_{\theta_{1}=0}^{\theta_{2}=2 \pi} \int_{\varphi_{1}}^{\varphi_{2}}\left[K_{n, m}^{\left(\alpha_{j}\right)}\left(\rho_{0}, \varphi, B_{j_{q}}(\theta)\right) \times \partial_{\rho} \tau_{\mathrm{FE}}\left(\rho_{0}, \varphi, \theta\right)-\tau_{\mathrm{FE}}\left(\rho_{0}, \varphi, \theta\right) \times \partial_{\rho} K_{n, m}^{\left(\alpha_{j}\right)}\left(\rho_{0}, \varphi, B_{j_{q}}(\theta)\right)\right] \\
& \times \rho_{0} R\left(1+\frac{\rho_{0}}{R} \cos \varphi\right) d \varphi d \theta \\
= & \pi \frac{\varphi_{2}-\varphi_{1}}{2} \int_{-1}^{1} \int_{-1}^{1}\left[K_{n, m}^{\left(\alpha_{j}\right)}\left(\rho_{0}, \varphi(\xi), B_{j_{q}}(\theta(\eta))\right) \times \partial_{\rho} \tau_{\mathrm{FE}}\left(\rho_{0}, \varphi(\xi), \theta(\eta)\right)\right. \\
& \left.-\tau_{\mathrm{FE}}\left(\rho_{0}, \varphi(\xi), \theta(\eta)\right) \times \partial_{\rho} K_{n, m}^{\left(\alpha_{j}\right)}\left(\rho_{0}, \varphi(\xi), B_{j_{q}}(\theta(\eta))\right)\right] \times \rho_{0} R\left(1+\frac{\rho_{0}}{R} \cos (\varphi(\xi))\right) d \xi d \eta \\
= & \pi \frac{\varphi_{2}-\varphi}{2} \sum_{j=1}^{n_{\mathrm{GP}}} \sum_{i=1}^{n_{\mathrm{GP}}} W_{j} W_{i}\left[K_{n, m}^{\left(\alpha_{j}\right)}\left(\rho_{0}, \varphi\left(\xi_{i}\right), B_{j_{q}}\left(\theta\left(\eta_{j}\right)\right)\right) \times \partial_{\rho} \tau_{\mathrm{FE}}\left(\rho_{0}, \varphi\left(\xi_{i}\right), \theta\left(\eta_{j}\right)\right)\right. \\
& \left.-\tau_{\mathrm{FE}}\left(\rho_{0}, \varphi\left(\xi_{i}\right), \theta\left(\eta_{j}\right)\right) \times \partial_{\rho} K_{n, m}^{\left(\alpha_{j}\right)}\left(\rho_{0}, \varphi\left(\xi_{i}\right), B_{j_{q}}\left(\theta\left(\eta_{j}\right)\right)\right)\right] \times \rho_{0} R\left(1+\frac{\rho_{0}}{R} \cos \left(\varphi\left(\xi_{i}\right)\right)\right)
\end{aligned}
$$


with $\varphi\left(\xi_{i}\right)$ computed by (92), and $\theta\left(\eta_{j}\right)=\frac{\theta_{2}-\theta_{1}}{2} \eta_{j}+\frac{\theta_{2}+\theta_{1}}{2}=\pi\left(\eta_{j}+1\right)$.

5.4.1. A 3-D torus with a circular crack. Consider the simple case of an inner cracked torus discussed in subsection 4.3.2 and showed in Figure 4. Here, a 3-D FE model is constructed as shown in Fig 11.

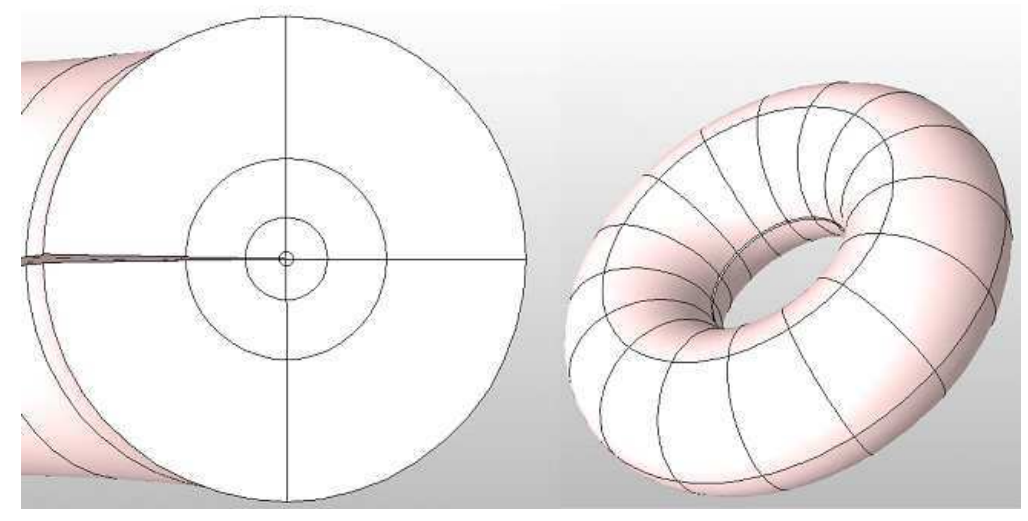

FIGURE 11. The 3-D FE model and the mesh.

First we check a simple axisymmetric case by applying the axisymmetric crack solution (61) on the torus's outer boundary with $A_{1}(\theta)=1, A_{2}(\theta)=A_{3}(\theta)=\cdots=0$ and $R=1$. We extract the first nine terms of $\tilde{A}_{1}(\theta)$, expecting that it is independent of $\theta$. The integral $J_{\rho_{0}}$ is computed by a Gauss quadrature with $n_{\mathrm{GP}}=90$ and using the FE solution at $p=8$ having a relative error in energy norm of $1.32 \%$.

In Table 7 we summarize the results of the computation $J_{\rho_{0}=0.5}\left[\tau_{\mathrm{FE}}, K_{n, m}^{(1 / 2)}\left[B_{1_{q}}\right]\right]$ for various $n \& m$. As

TABLE 7. Extracted $a_{1_{q}}$ for different $m$ and $n$ in the case of a circular crack with homogeneous Neumann BCs

\begin{tabular}{|l||c|c|c|c|c|c|c|c|c|}
\hline \multicolumn{1}{|c||}{} & \multicolumn{8}{c|}{ Extracted $a_{1_{q}}$ by $J_{\rho_{0}=1 / 2}\left[\tau, K_{n, m}^{(1 / 2)}\left[B_{1_{q}}\right]\right]$} \\
\cline { 2 - 10 } & $a_{1_{0}}$ & $a_{1_{1}}$ & $a_{1_{2}}$ & $a_{1_{3}}$ & $a_{1_{4}}$ & $a_{1_{5}}$ & $a_{1_{6}}$ & $a_{1_{7}}$ & $a_{1_{8}}$ \\
\hline \hline$m=n=0$ & 0.99768 & $-1.78 \mathrm{E}-06$ & $-3.36 \mathrm{E}-11$ & $-1.08 \mathrm{E}-06$ & $4.29 \mathrm{E}-12$ & $1.20 \mathrm{E}-05$ & $4.60 \mathrm{E}-11$ & $-1.78 \mathrm{E}-03$ & $-7.73 \mathrm{E}-11$ \\
\hline$m=n=2$ & 1.00005 & $-8.22 \mathrm{E}-07$ & $-3.83 \mathrm{E}-11$ & $7.69 \mathrm{E}-07$ & $6.77 \mathrm{E}-12$ & $-3.05 \mathrm{E}-06$ & $1.08 \mathrm{E}-10$ & $2.49 \mathrm{E}-03$ & $-2.65 \mathrm{E}-10$ \\
\hline$m=n=4$ & 0.99988 & $-5.02 \mathrm{E}-07$ & $-3.87 \mathrm{E}-11$ & $1.71 \mathrm{E}-06$ & $7.17 \mathrm{E}-12$ & $-1.43 \mathrm{E}-05$ & $1.29 \mathrm{E}-10$ & $1.02 \mathrm{E}-02$ & $-3.65 \mathrm{E}-10$ \\
\hline
\end{tabular}

expected, the extracted $\tilde{A}_{1}(\theta)$ is independent of $\theta$ and nicely converged to 1 .

Next, we consider a nonaxisymmetric case for which

$$
A_{1}(\theta)=1+2 \cos \theta+3 \sin \theta+4 \cos 2 \theta+5 \sin 2 \theta+6 \cos 3 \theta+7 \sin 3 \theta+8 \cos 4 \theta+9 \sin 4 \theta
$$

and $A_{0}=A_{2}(\theta)=A_{3}(\theta)=\cdots=0$. Applying on the outer surface of the torus the solution (108) with the single nonzero $A_{1}(\theta)$, the FE solution is obtained at $p=8$ with a relative error in the energy norm of $0.98 \%$. In Table 8 we present the $\%$ relative error in $a_{1_{q}}$ extracted by $J_{\rho_{0}=1 / 4}\left[\tau_{\mathrm{FE}}, K_{0,0}^{(1 / 2)}\left[B_{1_{q}}\right]\right]$ for different quadrature orders $n_{\mathrm{GP}}$.

One may notice that beyond 32 integration points the integration error is already negligible.

Convergence of extracted $a_{j_{q}}$ for different $n$ and $m$ :

We compute $J_{\rho_{0}=1 / 4}\left[\tau_{\mathrm{FE}}, K_{n, m}^{(1 / 2)}\left[B_{1_{q}}\right]\right]$ with $n_{\mathrm{GP}}=32$ for different $n$ and $m$ and summarize the results in Table 9.

The error in extracted $a_{1_{q}}$ does not decrease if $m$ is not high enough in the extraction function $K_{n, m}^{\left(\alpha_{j}\right)}$. 
TABLE 8. \% Relative error in extracted $a_{1_{q}}$ for different Gauss quadrature orders in the case of a circular crack with homogeneous Neumann BCs

\begin{tabular}{|c||c|c|c|c|c|c|c|c|c|}
\hline \multicolumn{1}{|c||}{} & \multicolumn{8}{c|}{ \% Relative error in extracted $a_{1_{q}}$ by $J_{\rho_{0}=1 / 4}\left[\tau, K_{0,0}^{(1 / 2)}\left[B_{1_{q}}\right]\right]$} \\
\cline { 2 - 10 } & $a_{1_{0}}$ & $a_{1_{1}}$ & $a_{1_{2}}$ & $a_{1_{3}}$ & $a_{1_{4}}$ & $a_{1_{5}}$ & $a_{1_{6}}$ & $a_{1_{7}}$ & $a_{1_{8}}$ \\
\hline \hline$n_{\mathrm{GP}}=10$ & 4.35 & 71.79 & 23.09 & 146.83 & 280.13 & 603.73 & 175.98 & 351.58 & 546.34 \\
\hline$n_{\mathrm{GP}}=15$ & 0.18 & 17.50 & 17.87 & 78.42 & 78.18 & 204.54 & 207.11 & 464.26 & 437.55 \\
\hline$n_{\mathrm{GP}}=32$ & 0.25 & 17.65 & 17.60 & 78.24 & 78.25 & 205.85 & 205.84 & 450.87 & 450.86 \\
\hline$n_{\mathrm{GP}}=54$ & 0.23 & 17.61 & 17.61 & 78.24 & 78.25 & 205.85 & 205.85 & 450.87 & 450.88 \\
\hline$n_{\mathrm{GP}}=90$ & 0.22 & 17.62 & 17.61 & 78.25 & 78.24 & 205.84 & 205.84 & 450.86 & 450.87 \\
\hline
\end{tabular}

TABLE 9. \% Relative error in extracted $a_{1_{q}}$ for different $n$ and $m$ in the case of a circular crack with homogeneous Neumann BCs

\begin{tabular}{|cc||c|c|c|c|c|c|c|c|c|}
\hline \multicolumn{1}{|c||}{} & \multicolumn{8}{c|}{ \% Relative error in extracted $a_{1_{q}}$ by $J_{\rho_{0}=1}\left[\tau, K_{n, m}^{(1 / 2)}\left[B_{1_{q}}\right]\right]$} \\
\cline { 4 - 11 } & $a_{1_{0}}$ & $a_{1_{1}}$ & $a_{1_{2}}$ & $a_{1_{3}}$ & $a_{1_{4}}$ & $a_{1_{5}}$ & $a_{1_{6}}$ & $a_{1_{7}}$ & $a_{1_{8}}$ \\
\hline \hline$m=0$ & $n=0$ & 0.004 & 3.610 & 3.593 & 14.670 & 14.671 & 33.921 & 33.920 & 62.495 & 62.491 \\
\hline$m=0$ & $n=2$ & 0.023 & 3.659 & 3.642 & 14.787 & 14.789 & 34.161 & 34.160 & 62.920 & 62.917 \\
\hline$m=0$ & $n=4$ & 0.023 & 3.659 & 3.642 & 14.787 & 14.789 & 34.161 & 34.160 & 62.921 & 62.917 \\
\hline$m=2$ & $n=0$ & 0.004 & 0.939 & 0.922 & 4.528 & 4.530 & 13.321 & 13.321 & 32.056 & 32.051 \\
\hline$m=2$ & $n=2$ & 0.023 & 0.166 & 0.148 & 1.382 & 1.385 & 6.345 & 6.345 & 20.090 & 20.084 \\
\hline$m=2$ & $n=4$ & 0.023 & 0.095 & 0.077 & 1.092 & 1.095 & 5.675 & 5.674 & 18.858 & 18.852 \\
\hline$m=4$ & $n=0$ & 0.004 & 0.894 & 0.877 & 3.807 & 3.809 & 9.624 & 9.624 & 20.212 & 20.207 \\
\hline$m=4$ & $n=2$ & 0.023 & 0.102 & 0.084 & 0.345 & 0.348 & 1.004 & 1.004 & 2.868 & 2.862 \\
\hline$m=4$ & $n=4$ & 0.023 & 0.028 & 0.011 & 0.016 & 0.019 & 0.127 & 0.126 & 0.946 & 0.940 \\
\hline
\end{tabular}

As an example, we also extracted $a_{30}$ which is the constant value associated with $A_{3}(\theta)$ so to make sure that it is practically zero. Indeed the extracted value is $a_{3_{0}}=10^{-4}$.

Convergence as a function of $\rho_{0}$ :

We fix $n=m=4$ and compute $J_{\rho_{0}}\left[\tau_{\mathrm{FE}}, K_{4,4}^{(1 / 2)}\left[B_{1_{q}}\right]\right]$ with $n_{\mathrm{GP}}=32$ for different $\rho_{0} \mathrm{~s}$ and summarize the results in Table 10 .

TABLE 10. $\%$ Relative error in extracted $a_{1_{q}}$ for different path radii $\rho_{0}$ in the case of a circular crack with homogeneous Neumann BCs.

\begin{tabular}{|c||c|c|c|c|c|c|c|c|c|}
\hline \multicolumn{1}{|c||}{} & \multicolumn{8}{c|}{ \% Relative error in extracted $a_{1_{q}}$ by $J_{\rho_{0}}\left[\tau, K_{4,4}^{(1 / 2)}\left[B_{1_{q}}\right]\right]$} \\
\cline { 2 - 10 } & $a_{1_{0}}$ & $a_{1_{1}}$ & $a_{1_{2}}$ & $a_{1_{3}}$ & $a_{1_{4}}$ & $a_{1_{5}}$ & $a_{1_{6}}$ & $a_{1_{7}}$ & $a_{1_{8}}$ \\
\hline \hline$\rho_{0}=0.5$ & 0.014 & 1.033 & 0.985 & 9.463 & 9.496 & 71.832 & 71.813 & 433.227 & 433.180 \\
\hline$\rho_{0}=0.4$ & 0.011 & 0.206 & 0.180 & 1.462 & 1.468 & 10.004 & 10.001 & 54.463 & 54.450 \\
\hline$\rho_{0}=0.3$ & 0.018 & 0.039 & 0.035 & 0.142 & 0.138 & 0.914 & 0.914 & 5.003 & 5.003 \\
\hline$\rho_{0}=0.2$ & 0.013 & 0.011 & 0.012 & 0.017 & 0.019 & 0.101 & 0.101 & 0.205 & 0.206 \\
\hline$\rho_{0}=0.1$ & 0.014 & 0.008 & 0.007 & 0.026 & 0.030 & 0.160 & 0.162 & 0.511 & 0.511 \\
\hline
\end{tabular}

As expected, the error in the extracted $a_{1_{q}}$ decreases as $\rho_{0}$ decreases.

The numerical experiments confirm that the convergence rates of the extracted $a_{1_{q}}^{\mathrm{FE}}$ match the convergence rates according to the estimates in (35). 
One may also notice that the relative error in the extracted $a_{j_{q}}$ by $J_{\rho_{0}=0.5}\left[\tau_{\mathrm{FE}}, K_{4,0}^{\left(\alpha_{j}\right)}\left[B_{j_{q}}\right]\right]$ is smaller than the relative error in energy norm.

5.4.2. A general problem: a penny shaped crack in a cylinder. Consider again the cylinder with a penny shaped crack shown in Figure 8 with Dirichlet boundary conditions on its outer surface and homogeneous Neumann BCs on crack surfaces. Specifically, the following BCs are applied:

$$
\begin{aligned}
\tau_{1}\left(r, x_{3}=1, \theta\right) & =r \theta^{2}(\theta-2 \pi)^{2} & & \text { on the upper surface } \\
\tau_{2}\left(r, x_{3}=-1, \theta\right) & =-r \theta^{2}(\theta-2 \pi)^{2} & & \text { on the lower surface } \\
\tau_{3}\left(r=2, x_{3}, \theta\right) & =0 & & \text { on the outer surface }
\end{aligned}
$$

Because the BCs (123)-(125) are $\theta$-depend a 3-D FE model is constructed as shown in Fig. 12.

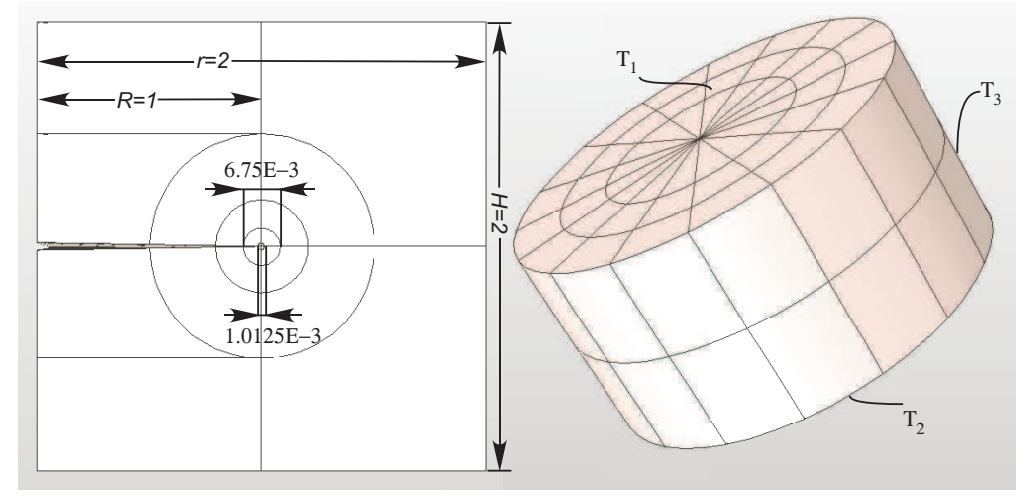

FIGURE 12. A penny shaped crack in a cylinder: 3-D FE model and a slice showing the crack.

Because an analytic solution to this problem is not available, we compute the first GEFIF $A_{1}$ (the most important one because it is associated with the singular flux) by the QDFM and also use the CIM [1] implemented in the commercial FE code to obtain pointwise values of $A_{1}$ along the circular singular edge. For the CIM the extraction path radius is 0.0035 , thus requiring a refined mesh in the vicinity of the crack edge. The FE relative error in energy norm is $1.33 \%$.

Extracted $a_{1_{q}}$ by $J_{\rho_{0}}\left[\tau_{\mathrm{FE}}, K_{4,4}^{(1 / 2)}\left[B_{1_{q}}\right]\right]$ for $\rho=0.35$ and $\rho_{0}=1 / 4$ using a Gauss quadrature of order 32 are given in Table 11. One may notice the fast convergence of the extracted coefficients $a_{1_{q}}$ as $q$ increases.

TABLE 11. Extracted $a_{1_{q}}$ for extracted at $\rho_{0}=0.35,0.25$ for a cylinder with a penny shaped crack with homogeneous Neumann BCs.

\begin{tabular}{|c||c|c|c|c|c|c|c|c|c|}
\hline \multicolumn{1}{|c||}{} & \multicolumn{10}{c|}{ Extracted $a_{1_{q}}$ by $J_{\rho_{0}}\left[\tau, K_{4,4}^{(1 / 2)}\left(B_{1_{q}}\right)\right]$} \\
\cline { 2 - 9 } & $a_{1_{0}}$ & $a_{1_{1}}$ & $a_{1_{2}}$ & $a_{1_{3}}$ & $a_{1_{4}}$ & $a_{1_{5}}$ & $a_{1_{6}}$ & $a_{1_{7}}$ & $a_{1_{8}}$ \\
\hline \hline$\rho_{0}=0.35$ & 44.0984 & -27.6679 & $7.249 \mathrm{E}-09$ & -0.9867 & $1.0735 \mathrm{E}-08$ & -0.1028 & $8.8255 \mathrm{E}-09$ & $-1.7016 \mathrm{E}-02$ & $-6.2489 \mathrm{E}-09$ \\
\hline$\rho_{0}=0.25$ & 44.0975 & -27.6584 & $7.038 \mathrm{E}-09$ & -0.9845 & $-4.7541 \mathrm{E}-09$ & -0.1015 & $1.2545 \mathrm{E}-09$ & $-1.5772 \mathrm{E}-02$ & $-3.6742 \mathrm{E}-09$ \\
\hline
\end{tabular}

The extracted $a_{1_{q}}$ are used in (103) to compute $\tilde{A}_{1}(\theta)$ which is shown in Fig 13 as a smooth curve compared to the pointwise values extracted by the CIM at $\rho_{0}=0.0035$.

One observes that the extracted $\tilde{A}_{1}(\theta)$ at $\rho_{0}=0.25$ and at $\rho_{0}=0.35$ by the QDFM match closely each other and the pointwise values extract by the CIM at $\rho_{0}=0.0035$. This demonstrates that the QDFM does not require a refined mesh in the vicinity of a circular crack tip which is a major advantage in 3-D domains, and at the same time produces a smooth and continuous GEFIF along the crack edge. 


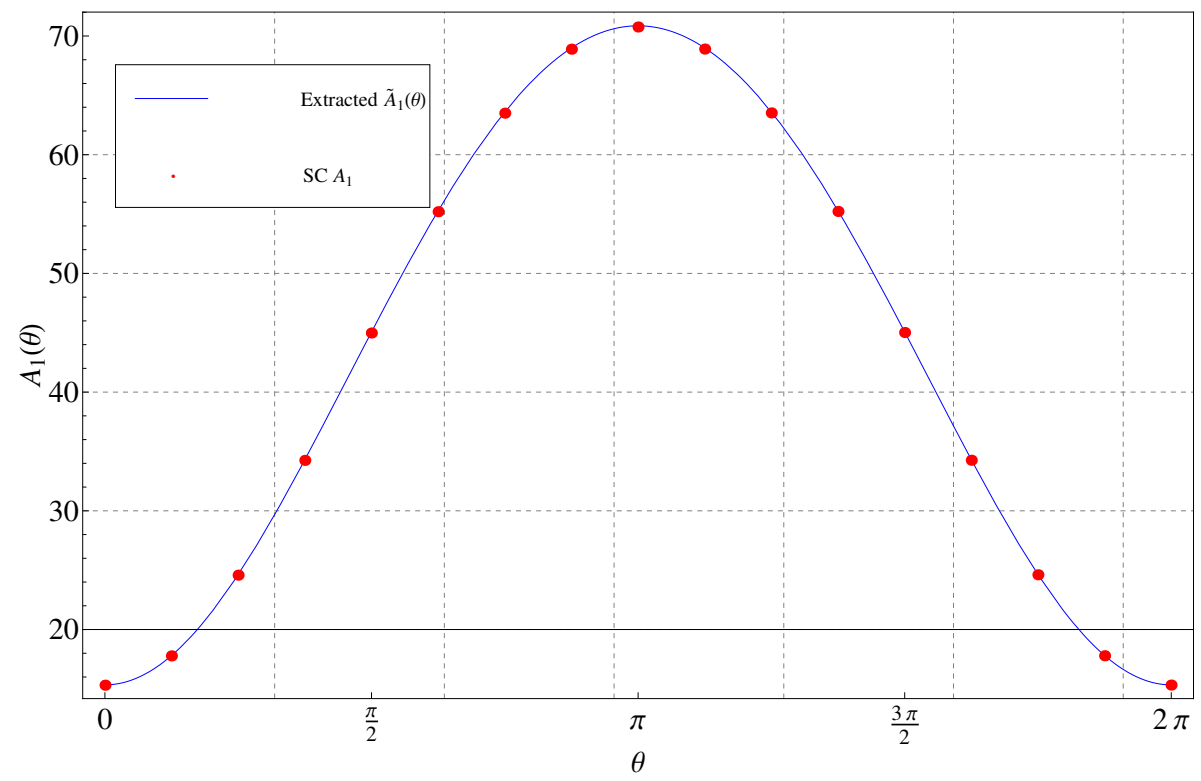

FIGURE 13. Extracted $\tilde{A}_{1}(\theta)$ by $J_{\rho_{0}=1 / 4}\left[\tau, K_{4,4}^{(1 / 2)}\left[B_{1_{q}}\right]\right]$ at $\rho_{0}=1 / 4$ and by the CIM at $\rho_{0}=0.0035$.

\section{SUMMARY AND CONCLUSIONS}

We extended the QDFM for extracting generalized edge flux intensity functions in the vicinity of a circular singular edge for axisymmetric as well as non-axisymmetric solutions. The formulation and performance of the method is demonstrated on the simplified Laplace equation. Although the QDFM is a mildly-surface-dependent integral, we demonstrated that by using a proper quasi-dual function $K_{n, m}^{\left(\alpha_{j}\right)}$ and proper extraction functions $B_{j}(\theta)$, one may extract the functional representation of the GEFIFs $A_{k}(\theta)$ accurately and efficiently.

Because in general only a FE approximation or the solution is available, we demonstrated that the QDFM in conjunction with $p$-FE methods provide highly accurate GEFIFs. These were obtained as a function along the circular edge, and are much more accurate compared to the FE solution (superconvergence property). Another major advantage is the possibility to use the FE solution on a torus away from the singular edge, thus it is not necessary to have a refined FE mesh in the vicinity of the singularity (which is a complicated and tedious task in 3-D domains).

Following the successful formulation of the QDFM and its efficiency it is extended to the elasticity set of equations following the same methodology presented herein. A straight-forward extension is possible with only technical challenges but no conceptual difficulties.

Acknowledgements: The first two authors gratefully acknowledge the support of this work by the Israel Science Foundation (grant No. 444/10).

\section{REFERENCES}

[1] I. Babuška and A. Miller. The post-processing approach in the finite element method - Part 2: The calculation of stress intensity factors. Int. Jour. Numer. Meth. Engrg., 20:1111-1129, 1984.

[2] H. Blum and M. Dobrowolski. On finite element methods for elliptic equations on domains with corners. Computing, 28:53-63, 1982. 
[3] P. Ciarlet, Jr., B. Jung, S. Kaddouri, S. Labrunie, and J. Zou. The Fourier singular complement method for the Poisson problem. II. Axisymmetric domains. Numer. Math., 102(4):583-610, 2006.

[4] M. Costabel, M. Dauge, and R. Duduchava. Asymptotics without logarithmic terms for crack problems. Communication in PDEs, 28(5-6):869-926, 2003.

[5] M. Costabel, M. Dauge, and Z. Yosibash. A quasidual function method for extracting edge stress intensity functions. SIAM Jour. Math. Anal., 35(5):1177-1202, 2004.

[6] M. Dauge. Elliptic boundary value problems in corner domains - smoothness and asymptotics of solutions. Lecture notes in Mathematics 1341, Springer-Verlag, Heidelberg, 1988.

[7] P. Grisvard. Elliptic problems in nonsmooth domains. Pitman Publishing, England, 1985.

[8] G. R. Irwin. Onset of fast crack propagation in high strength steel and aluminum alloys. In Sagamore Research Conference Proceedings - Vol 2, pages 289-305, 1956.

[9] G. R. Irwin. Analysis of stresses and strains near the end of a crack transversing a plate. Trans. ASME, Jour. Appl. Mech., 24:361364, 1957.

[10] V. G. Maz’ya and J. Rossmann. Über die Asymptotik der Lösungen elliptischer Randwertaufgaben in der Umgebung von Kanten. Math. Nachr. 138:27-53, 1988.

[11] N. Omer, Z. Yosibash, M. Costabel, and M. Dauge. Edge flux intensity functions in polyhedral domains and their extraction by a quasidual function method. Int. Jour. Fracture, 129:97-130, 2004.

[12] S. Shannon, Z. Yosibash, M. Dauge, and M. Costabel. Primal and Shadow functions, Dual and Dual-Shadow functions for a circular crack and a circular $90^{\circ}$ V-notch with Neumann boundary conditions. Research report, 2012. hal.archives-ouvertes.fr/hal-00765619

[13] I. N. Sneddon. The distribution of stress in the neighbourhood of a crack in an elastic solid. Proc. Royal Soc. London. Series A. Math. Physical Sci., 187:229-260, 1946.

[14] Z. Yosibash, N. Omer, M. Costabel, and M. Dauge. Edge stress intensity functions in polyhedral domains and their extraction by a quasidual function method. Int. Jour. Fracture, 136:37 - 73, 2005.

[15] Z. Yosibash, S. Shannon, M. Dauge, and M. Costabel. Circular edge singularities for the Laplace equation and the elasticity system in 3-D domains. Int. Jour. Fracture, 168:31-52, 2011. 
APPENDiX A. AN EXPLICIT EXPRESSION FOR $K_{\infty, 0}^{(1 / 2)}$ FOR A CIRCUlAR CRACK

If we evaluate the successive ratios of the $\beta_{i}$ 's in Table 1 we find a simple hypergeometric law.

$\begin{array}{cccccccccc}\frac{\beta_{1}}{\beta_{0}} & \frac{\beta_{2}}{\beta_{1}} & \frac{\beta_{3}}{\beta_{2}} & \frac{\beta_{4}}{\beta_{3}} & \frac{\beta_{5}}{\beta_{4}} & \frac{\beta_{6}}{\beta_{5}} & \frac{\beta_{7}}{\beta_{6}} & \frac{\beta_{8}}{\beta_{7}} & \frac{\beta_{9}}{\beta_{8}} & \frac{\beta_{10}}{\beta_{9}} \\ \frac{1}{4} & \frac{3}{8} & \frac{5}{12} & \frac{7}{16} & \frac{9}{20} & \frac{11}{24} & \frac{13}{28} & \frac{15}{32} & \frac{17}{36} & \frac{19}{40}\end{array}$

TABLE 12. Successive ratios between coefficients in the expansion (65) of the QDF $K_{n, 0}^{(1 / 2)}$.

One can prove that the sequence $K_{n, 0}^{(1 / 2)}\left[B_{1}\right]$ is converging and compute its limit, as follows. Setting $Z=$ $\rho e^{\imath \varphi}$, we have

Hence

$$
\rho^{i+1 / 2} \sin \frac{(2 i+1) \varphi}{2}=\Im Z^{i+1 / 2}, \quad i=0,1, \ldots
$$

$$
K_{n, 0}^{(1 / 2)}=\frac{1}{2 \pi^{2} \rho R} \Im\left(Z^{1 / 2}\left[\sum_{i=0}^{n}(-1)^{i} \beta_{i}\left(\frac{Z}{R}\right)^{i}\right]\right)
$$

and since $\frac{\beta_{i+1}}{\beta_{i}}=\frac{2 i+1}{2(2 i+2)}$ (Table 12), we find the limit

$$
K_{\infty, 0}^{(1 / 2)}=\frac{1}{2 \pi^{2} \rho R} \Im\left(Z^{1 / 2}\left(1+\frac{Z}{2 R}\right)^{-1 / 2}\right) .
$$


APPENDiX B. TABles With RESUltS OF THE SURFACE INTEGRAL IN A NON-AXISYMMETRIC CASE

TABLE 13. The remainder of $J_{\rho_{0}}\left[\tau, K_{n, m}^{(1 / 2)}\left[B_{1_{0}}\right]\right]$

\begin{tabular}{lccc}
\hline \multicolumn{3}{c}{$B_{1_{0}}(\theta)=b_{1_{0}}=\frac{1}{2 \pi^{2} R}$} \\
\hline$n=0$ & $a_{3_{0}} \mathcal{O}\left\{\rho_{0}\left(\frac{\rho_{0}}{R}\right)\right\}$ & $a_{3_{0}} \mathcal{O}\left\{\rho_{0}\left(\frac{\rho_{0}}{R}\right)\right\}$ & $a_{3_{0}} \mathcal{O}\left\{\rho_{0}\left(\frac{\rho_{0}}{R}\right)\right\}$ \\
$n=1$ & $a_{5_{0}} \mathcal{O}\left\{\rho_{0}^{2}\left(\frac{\rho_{0}}{R}\right)^{2}\right\}$ & $a_{5_{0}} \mathcal{O}\left\{\rho_{0}^{2}\left(\frac{\rho_{0}}{R}\right)^{2}\right\}$ & $a_{5_{0}} \mathcal{O}\left\{\rho_{0}^{2}\left(\frac{\rho_{0}}{R}\right)^{2}\right\}$ \\
$n=2$ & $a_{7_{0}} \mathcal{O}\left\{\rho_{0}^{3}\left(\frac{\rho_{0}}{R}\right)^{3}\right\}$ & $a_{7_{0}} \mathcal{O}\left\{\rho_{0}^{3}\left(\frac{\rho_{0}}{R}\right)^{3}\right\}$ & $a_{7_{0}} \mathcal{O}\left\{\rho_{0}^{3}\left(\frac{\rho_{0}}{R}\right)^{3}\right\}$ \\
$n=3$ & $a_{9_{0}} \mathcal{O}\left\{\rho_{0}^{4}\left(\frac{\rho_{0}}{R}\right)^{4}\right\}$ & $a_{9_{0}} \mathcal{O}\left\{\rho_{0}^{4}\left(\frac{\rho_{0}}{R}\right)^{4}\right\}$ & $a_{9_{0}} \mathcal{O}\left\{\rho_{0}^{4}\left(\frac{\rho_{0}}{R}\right)^{4}\right\}$ \\
$n=4$ & $a_{11_{0}} \mathcal{O}\left\{\rho_{0}^{5}\left(\frac{\rho_{0}}{R}\right)^{5}\right\}$ & $a_{11_{0}} \mathcal{O}\left\{\rho_{0}^{5}\left(\frac{\rho_{0}}{R}\right)^{5}\right\}$ & $a_{11_{0}} \mathcal{O}\left\{\rho_{0}^{5}\left(\frac{\rho_{0}}{R}\right)^{5}\right\}$ \\
\hline
\end{tabular}

TABLE 14. The remainder of $J_{\rho_{0}}\left[\tau, K_{n, m}^{(1 / 2)}\left[B_{1_{1}}\right]\right]$

\begin{tabular}{|c|c|c|c|}
\hline \multicolumn{4}{|c|}{$B_{1_{1}}(\theta)=b_{1_{1}} \cos (\theta)=\frac{1}{\pi^{2} R} \cos (\theta)$} \\
\hline & $m=0$ & $m=2$ & $m=4$ \\
\hline$n=0$ & $a_{1_{1}} \mathcal{O}\left\{\left(\frac{\rho_{0}}{R}\right)^{2}\right\}+a_{3_{1}} \mathcal{O}\left\{\rho_{0}\left(\frac{\rho_{0}}{R}\right)\right\}$ & $a_{1_{1}} \mathcal{O}\left\{\left(\frac{\rho_{0}}{R}\right)^{2}\right\}+a_{3_{1}} \mathcal{O}\left\{\rho_{0}\left(\frac{\rho_{0}}{R}\right)\right\}$ & $a_{1_{1}} \mathcal{O}\left\{\left(\frac{\rho_{0}}{R}\right)^{2}\right\}+a_{3_{1}} \mathcal{O}\left\{\rho_{0}\left(\frac{\rho_{0}}{R}\right)\right\}$ \\
\hline$n=1$ & $a_{1_{1}} \mathcal{O}\left\{\left(\frac{\rho_{0}}{R}\right)^{2}\right\}$ & $a_{1_{1}} \mathcal{O}\left\{\left(\frac{\rho_{0}}{R}\right)^{4}\right\}+a_{5_{1}} \mathcal{O}\left\{\rho_{0}^{2}\left(\frac{\rho_{0}}{R}\right)^{2}\right\}$ & $a_{1_{1}} \mathcal{O}\left\{\left(\frac{\rho_{0}}{R}\right)^{4}\right\}+a_{5_{1}} \mathcal{O}\left\{\rho_{0}^{2}\left(\frac{\rho_{0}}{R}\right)^{2}\right\}$ \\
\hline$n=2$ & $a_{1_{1}} \mathcal{O}\left\{\left(\frac{\rho_{0}}{R}\right)^{2}\right\}$ & $a_{1_{1}} \mathcal{O}\left\{\left(\frac{\rho_{0}}{R}\right)^{4}\right\}$ & $a_{1_{1}} \mathcal{O}\left\{\left(\frac{\rho_{0}}{R}\right)^{5}\right\}$ \\
\hline$n=3$ & $a_{1_{1}} \mathcal{O}\left\{\left(\frac{\rho_{0}}{R}\right)^{2}\right\}$ & $a_{1_{1}} \mathcal{O}\left\{\left(\frac{\rho_{0}}{R}\right)^{4}\right\}$ & $a_{1_{1}} \mathcal{O}\left\{\left(\frac{\rho_{0}}{R}\right)^{6}\right\}$ \\
\hline$n=4$ & $a_{1_{1}} \mathcal{O}\left\{\left(\frac{\rho_{0}}{R}\right)^{2}\right\}$ & $a_{1_{1}} \mathcal{O}\left\{\left(\frac{\rho_{0}}{R}\right)^{4}\right\}$ & $a_{1_{1}} \mathcal{O}\left\{\left(\frac{\rho_{0}}{R}\right)^{6}\right\}$ \\
\hline
\end{tabular}

TABLE 15 . The remainder of $J_{\rho_{0}}\left[\tau, K_{n, m}^{(1 / 2)}\left[B_{1_{3}}\right]\right]$

\begin{tabular}{|c|c|c|c|}
\hline \multicolumn{4}{|c|}{$B_{1_{3}}(\theta)=b_{1_{3}} \cos (\theta)=\frac{1}{\pi^{2} R} \cos (2 \theta)$} \\
\hline & $m=0$ & $m=2$ & $m=4$ \\
\hline$n=1$ & $a_{1_{3}} \mathcal{O}\left\{\left(\frac{\rho_{0}}{R}\right)^{2}\right\}+a_{3_{3}} \mathcal{O}\left\{\rho_{0}\left(\frac{\rho_{0}}{R}\right)\right\}$ & $a_{1_{3}} \mathcal{O}\left\{\left(\frac{\rho_{0}}{R}\right)^{2}\right\}+a_{3_{3}} \mathcal{O}\left\{\rho_{0}\left(\frac{\rho_{0}}{R}\right)\right\}$ & $a_{1_{3}} \mathcal{O}\left\{\left(\frac{\rho_{0}}{R}\right)^{2}\right\}+a_{3_{3}} \mathcal{O}\left\{\rho_{0}\left(\frac{\rho_{0}}{R}\right)\right\}$ \\
\hline$n=1$ & $a_{1_{3}} \mathcal{O}\left\{\left(\frac{\rho_{0}}{R}\right)^{2}\right\}$ & $a_{1_{3}} \mathcal{O}\left\{\left(\frac{\rho_{0}}{R}\right)^{4}\right\}+a_{5_{3}} \mathcal{O}\left\{\rho_{0}^{2}\left(\frac{\rho_{0}}{R}\right)^{2}\right\}$ & $a_{1_{3}} \mathcal{O}\left\{\left(\frac{\rho_{0}}{R}\right)^{4}\right\}+a_{5_{3}} \mathcal{O}\left\{\rho_{0}^{2}\left(\frac{\rho_{0}}{R}\right)^{2}\right\}$ \\
\hline$n=2$ & $a_{1_{3}} \mathcal{O}\left\{\left(\frac{\rho_{0}}{R}\right)^{2}\right\}$ & $a_{1_{3}} \mathcal{O}\left\{\left(\frac{\rho_{0}}{R}\right)^{4}\right\}$ & $a_{1_{3}} \mathcal{O}\left\{\left(\frac{\rho_{0}}{R}\right)^{5}\right\}$ \\
\hline$n=$ & $a_{1_{3}} \mathcal{O}\left\{\left(\frac{\rho_{0}}{R}\right)^{2}\right\}$ & $a_{1_{3}} \mathcal{O}\left\{\left(\frac{\rho_{0}}{R}\right)^{4}\right\}$ & $a_{1_{3}} \mathcal{O}\left\{\left(\frac{\rho_{0}}{R}\right)^{6}\right\}$ \\
\hline$n=$ & $a_{1_{3}} \mathcal{O}\left\{\left(\frac{\rho_{0}}{R}\right)^{2}\right\}$ & $a_{1_{3}} \mathcal{O}\left\{\left(\frac{\rho_{0}}{R}\right)^{4}\right\}$ & $a_{1_{3}} \mathcal{O}\left\{\left(\frac{\rho_{0}}{R}\right)^{6}\right\}$ \\
\hline
\end{tabular}

Z.Y. \& S.S.: Pearlstone Center for Aeronautical Eng. Studies, Dept. of Mechanical Engineering, BenGurion University of the Negev, Beer-SheVa, 84105, Israel, Tel.: +972-8-6477103, FAX: +972-8-6477101

E-mail address: zohary@bgu.ac.il

M.D. \& M.C.: IRMAR, Université de Rennes 1, Campus de Beaulieu, 35042 Rennes Cedex, France E-mail address: monique.dauge@univ-rennes1.fr

E-mail address: martin.costabel@univ-rennesl.fr 